\title{
FROM RUIN TO BANKRUPTCY FOR COMPOUND POISSON SURPLUS PROCESSES
}

BY

HANSJÖRG ALBRECHER AND VOLKMAR LAUTSCHAM

\begin{abstract}
In classical risk theory, the infinite-time ruin probability of a surplus process $C_{t}$ is calculated as the probability of the process becoming negative at some point in time. In this paper, we consider a relaxation of the ruin concept to the concept of bankruptcy, according to which one has a positive surplus-dependent probability to continue despite temporary negative surplus. We study the resulting bankruptcy probability for the compound Poisson risk model with exponential claim sizes for different bankruptcy rate functions, deriving analytical results, upper and lower bounds as well as an efficient simulation method. Numerical examples are given and the results are compared with the classical ruin probabilities. Finally, it is illustrated how the analysis can be extended to study the discounted penalty function under this relaxed ruin criterion.
\end{abstract}

\section{KEYWORDS}

Classical risk process, Omega model, ruin probability, discounted penalty function, bankruptcy rate function.

\section{INTRODUCTION}

In classical risk theory, ruin of a company is defined as the event where some surplus process $C_{t}$ becomes negative for the first time. Conversely, using a bankruptcy concept, the entity would go bankrupt randomly for negative $C_{t}$ levels at some bankruptcy rate $\omega(\cdot)$, subject to no prior bankruptcy event. The idea of extending ruin to this more general bankruptcy concept was introduced in Albrecher et al. (2011) in a discussion around a company's equity value process described by its expected discounted dividends over time, with dividends being paid up to the time of bankruptcy whenever an underlying process exceeds some dividend barrier $b$.

This paper examines the bankruptcy idea for a surplus processes with jumps, and we will formulate the ideas for an insurance application although other 
interpretations will be possible. Consider a Cramér-Lundberg setup to describe the insurer's surplus $C_{t}$ at time $t$ as

$$
C_{t}=x+c t-S_{t}
$$

where $C_{0}=x \geq 0$ is the initial surplus, $c$ is the premium rate and $S_{t}$ is the aggregate claim amount up to time $t$ modeled as a compound Poisson random variable with intensity $\lambda$ and positive jump sizes with cumulative distribution function $F_{Y}$ (in most parts of the present paper, the jump size distribution will be assumed to be exponential, although more general distributions (such as phase-type) lead to structurally similar equations and solution strategies which can still yield explicit results, see the respective remarks in later sections). In classical ruin theory, the insurer goes out of business at the time of ruin $\tau_{\text {ruin }}=\inf \left\{t>0 \mid C_{t}<0\right\}$.

In this paper, it is assumed that the insurer may be allowed to continue the business despite temporary negative surplus. The approach taken here differs from models with absolute ruin, as for instance studied in Gerber (1971), Dassios and Embrechts (1989) and Zhu and Yang (2008) in that we take a fully probabilistic approach. Concretely, a suitable locally bounded bankruptcy rate function $\omega\left(C_{t}\right)$ depending on the size of the negative surplus is defined on $(-\infty, 0]$. Given some negative surplus $C_{s}<0$ and no prior bankruptcy event, the probability of bankruptcy on the time interval $[s, s+d t)$ is $\omega\left(C_{s}\right) d t$. We assume that $\omega(\cdot) \geq 0$ and $\omega(x) \geq \omega(y)$ for $|x| \geq|y|$ to reflect that the likelihood of bankruptcy does not decrease as the surplus becomes more negative. Let $\tau$ be the resulting time of bankruptcy, and define the overall probability of bankruptcy as

$$
\psi(x)=\mathbb{E}\left[\mathbb{1}_{\{\tau<\infty\}} \mid C_{0}=x\right]=\mathbb{P}\left[\tau<\infty \mid C_{0}=x\right] .
$$

Note that the classical ruin probability $\psi_{\text {ruin }}(x)$ is retained for the limit $\omega(y) \equiv$ $\infty$ for all $y<0$.

In general, the idea is that whenever the surplus level becomes negative, there may still be a chance to survive, and it is modeled that survival is less likely the lower such a negative surplus level is. Conceptually, the replacement of the ruin concept by bankruptcy first of all removes the binary feature of the classical framework where the surplus process survives at $x=0$, but is killed for arbitrarily small negative surplus levels $x=0^{-}$. From a practical viewpoint, this is underpinned by the fact that in many jurisdictions the regulator would take control as an insurer's financial situation deteriorates, and measures would be undertaken during a rehabilitation period with the aim of curing the insurer's financial problems. Only when such measures fail, the insurer will typically go into liquidation (cf. Schacht and Hepler, 2007; Insurance Information Institute, 2012). In particular, temporary financial support to bridge a period of negative surplus could come from a solvent parent company that fears reputational damage in case of liquidation of one of its subsidiaries, from insurance guarantee schemes (cf. Oxera, 2007), or from governments that consider the insurer too big to fail (which was observed on several occasions in the United States and 
Europe during the financial crisis starting in 2007). If corrective actions and the decision whether an insurance business can continue its operations are taken already before hitting negative surplus levels (for example, regulator intervention when a minimum surplus level $s$ is not obeyed), one can translate this situation into the shifted bankruptcy problem with initial capital $x-s$ and an adequate choice of $\omega(\cdot)$.

To add another possible interpretation, there is a conceptual connection to contingent capital arrangements (cf. Glasserman and Nouri, 2010; Maes and Schoutens, 2010; Chen et al., 2013), where financial service firms pre-arrange capital injections or guarantees that could be triggered by low or negative surplus levels. For example, choose $a>0$ and define $\omega(x)$ arbitrarily large for $x<-a$ and $\omega(x) \equiv \omega_{c}$ for $-a \leq x<0$. One could then interpret the connected bankruptcy model as an unfunded financial guarantee where the guarantor promises to pay open claims up to some level $a$ in case of liquidation of the insurer. There will be counterparty risk linked to the guarantee, and default of the guarantor would mean bankruptcy for the insurer at negative surplus levels. The rate $\omega(x)$ could be seen as a guarantor default rate in this case.

For the particular case of constant bankruptcy rate functions $\omega(x) \equiv \omega_{c}$, the bankruptcy concept is mathematically also linked to other recent results. In Albrecher et al. (2011b), a model was set up where the surplus level $C_{t}$ is only observed at discrete random observation times (lack of information), so that ruin does not occur automatically as soon as the surplus drops negative. For exponentially distributed observation times, those results for the corresponding ruin probability coincide with our bankruptcy probability for constant bankruptcy function $\omega_{c}$, see also Albrecher et al (2011a). Also, Landriault et al. (2011) generally discuss occupation times for spectrally negative Lévy processes. For exponential implementation delay rates $\omega_{c}$, the authors mention the link between occupation times and the probability of ruin as $1-\mathbb{P}\left[\tau_{\text {ruin }}<\infty\right]=$ $\mathbb{E}\left[\exp \left\{-\omega_{c} \int_{0}^{\infty} \mathbb{1}_{\left\{C_{t}<0\right\}} d t\right\}\right]$. Again, this result coincides with our result for a constant bankruptcy rate function $\omega_{c}$.

This paper is organized as follows. Section 2 derives equations for the probability of bankruptcy $\psi(x)$, which are solved explicitly for some simple choices of bankruptcy rate functions and exponential claim sizes. Since for general bankruptcy rate functions, exact expressions for $\psi(x)$ are hard to obtain, Section 2.2 demonstrates how piecewise constant bankruptcy rate functions can be efficiently used as approximations, and Section 3 illustrates numerically the accuracy of such approximations. An effective simulation technique is introduced and tested as well. Finally, Section 4 discusses extensions from the probability of bankruptcy to the discounted penalty function which allows to investigate additional risk measures, such as the shortfall at the time of bankruptcy. 


\section{THE PROBABILITY OF BANKRUPTCY}

For technical reasons, let us extend the definition of $\omega(x)$ to the entire real line by specifying $\omega(x)=0$ for $x \geq 0$. Conditioning on the first occurrence time of either a claim or an event of bankruptcy up to time $h>0$ yields for $x \geq 0$ that

$$
\psi(x)=e^{-\lambda h} \psi(x+c h)+\int_{0}^{h} \lambda e^{-\lambda t} \int_{0}^{\infty} \psi(x+c t-y) d F_{Y}(y) d t,
$$

and for $x<0$ that

$$
\begin{aligned}
\psi(x)= & e^{-\lambda h-\int_{0}^{h} \omega(x+c y) d y} \psi(x+c h)+\int_{0}^{h} e^{-\lambda t} \omega(x+c t) e^{-\int_{0}^{t} \omega(x+c y) d y} d t \\
& +\int_{0}^{h} e^{-\int_{0}^{t} \omega(x+c y) d y} \lambda e^{-\lambda t} \int_{0}^{\infty} \psi(x+c t-y) d F_{Y}(y) d t .
\end{aligned}
$$

Choosing $x=0$ in (3) and letting $h \rightarrow 0$ shows right-continuity of $\psi(x)$ in $x=0$, whereas choosing $x=-c h$ in (4) and letting $h \rightarrow 0$ provides leftcontinuity (recall that $\omega\left(0^{-}\right)$is by definition bounded), so that $\psi(x)$ is indeed continuous in $x=0$, i.e.

$$
\psi\left(0^{-}\right)=\psi\left(0^{+}\right) .
$$

By the same line of reasoning, one can see that $\psi(x)$ is continuous for all values of $x \in \mathbb{R}$. Differentiating (3) and (4) w.r.t. $h$ and taking the limit $h \rightarrow 0$ leads to

$$
\begin{aligned}
& x \geq 0: 0=c \psi_{+}^{\prime}(x)-\lambda \psi(x)+\lambda \int_{0}^{\infty} \psi(x-y) d F_{Y}(y), \\
& x<0: 0=c \psi_{+}^{\prime}(x)-(\lambda+\omega(x)) \psi(x)+\omega(x)+\lambda \int_{0}^{\infty} \psi(x-y) d F_{Y}(y),
\end{aligned}
$$

where $\psi_{+}^{\prime}(x)$ denotes the right-hand derivative of $\psi(x)$. Replacing $x$ by $x-c h$ in (3) and (4) for sufficiently small $h$, followed by a differentiation w.r.t. $h$ and $h \rightarrow 0$ accordingly yields

$$
\begin{aligned}
& x>0: 0=c \psi_{-}^{\prime}(x)-\lambda \psi(x)+\lambda \int_{0}^{\infty} \psi(x-y) d F_{Y}(y), \\
& x<0: 0=c \psi_{-}^{\prime}(x)-(\lambda+\omega(x)) \psi(x)+\omega(x)+\lambda \int_{0}^{\infty} \psi(x-y) d F_{Y}(y)
\end{aligned}
$$

for the left-hand derivative $\psi_{-}^{\prime}(x)$. By the continuity of $\psi(x)$ for all $x \in \mathbb{R}$, comparing (6) and (8) it is hence clear that the derivative $\psi^{\prime}(x)$ exists for all $x>$ 0 . Correspondingly, comparing (7) and (9) shows that for $x<0$ the derivative $\psi^{\prime}(x)$ exists whenever $\omega(x)$ is continuous. Furthermore, from (5), (6) and (9), one sees that

$$
\psi_{+}^{\prime}(0)-\psi_{-}^{\prime}\left(0^{-}\right)=\frac{1}{c}\left[\omega\left(0^{-}\right)\left(1-\psi\left(0^{-}\right)\right)\right],
$$


so that the derivative of $\psi(x)$ is continuous in $x=0$ if $\omega\left(0^{-}\right)=0$ (the other case $\psi\left(0^{-}\right)=1$ refers to the classical ruin situation with $\omega\left(0^{-}\right)=\infty$, which is not of interest here). For ease of notation, we will always write $\psi^{\prime}(x)$ for $x<0$ in the sequel with the understanding that this is to be interpreted as a one-sided derivative at discontinuity points of $\omega(x)$.

Let us divide $\psi(x)$ into an upper (' $u$ ') and a lower (' $l$ ') function depending on the value of $x$,

$$
\psi(x)=\left\{\begin{array}{ll}
\psi_{u}(x) & \text { for } x \geq 0 \\
\psi_{l}(x) & \text { for } x<0
\end{array},\right.
$$

to give

$$
\begin{aligned}
x \geq 0: 0= & c \psi_{u}^{\prime}(x)-\lambda \psi_{u}(x) \\
& +\lambda\left(\int_{0}^{x} \psi_{u}(x-y) d F_{Y}(y)+\int_{x}^{\infty} \psi_{l}(x-y) d F_{Y}(y)\right), \\
x<0: 0= & c \psi_{l}^{\prime}(x)-(\lambda+\omega(x)) \psi_{l}(x)+\omega(x) \\
& +\lambda \int_{0}^{\infty} \psi_{l}(x-y) d F_{Y}(y),
\end{aligned}
$$

with $\psi_{u}\left(0^{+}\right)=\psi_{l}\left(0^{-}\right)$and $\psi_{u}^{\prime}\left(0^{+}\right)-\psi_{l}^{\prime}\left(0^{-}\right)=\frac{1}{c}\left[\omega\left(0^{-}\right)\left(1-\psi_{l}\left(0^{-}\right)\right)\right]$. For simplicity, we will assume throughout the rest of the paper that the claim sizes are exponentially distributed $(Y \sim \operatorname{Exp}(v))$. In this case, the integrals can be eliminated by applying the operator $(d / d x+v)$ to (11) and (12), and one arrives at the following system of linear differential equations,

$$
\begin{aligned}
x \geq 0: 0 & =c \psi_{u}^{\prime \prime}(x)+(v c-\lambda) \psi_{u}^{\prime}(x), \\
x<0: 0= & c \psi_{l}^{\prime \prime}(x)+(v c-(\lambda+\omega(x))) \psi_{l}^{\prime}(x) \\
& -\left(\omega^{\prime}(x)+v \omega(x)\right) \psi_{l}(x)+\omega^{\prime}(x)+v \omega(x) .
\end{aligned}
$$

For $x \geq 0,(13)$ has constant coefficients and one can easily obtain

$$
\psi_{u}(x)=A e^{-\left(v-\frac{\lambda}{c}\right) x}+B,
$$

with constants $A, B \in \mathbb{R}$. By the net profit condition, one has $v-\frac{\lambda}{c}>0$, and from $\lim _{x \rightarrow \infty} \psi_{u}(x)=0$, it follows that $B=0$. Note that

$$
\psi_{\text {ruin }}(x)=\frac{\lambda}{v c} e^{-\left(\nu-\frac{\lambda}{c}\right) x}, \quad x \geq 0,
$$

in the classical case if claim sizes are exponentially distributed (see e.g. Gerber, 1971), which differs from (15) only by the constant $A$. Clearly, $\psi_{l}(x) \equiv 1$ is always a particular solution of (14), so that one can write

$$
\psi_{l}(x)=1+A_{l} \cdot h(x)
$$


where $A_{l}$ is some constant and $h(x)$ is the homogeneous solution of (14) that fulfills $h(-\infty)=0$. The latter condition ensures the natural requirement $\lim _{x \rightarrow-\infty} \psi_{l}(x)=1$. The continuity conditions (5) and (10) now give the equation system

$$
\begin{aligned}
A & =1+A_{l} \cdot h(0) \\
-\left(v-\frac{\lambda}{c}\right) A & =A_{l} \cdot h^{\prime}(0)+\frac{1}{c} \omega\left(0^{-}\right)\left(-A_{l} \cdot h(0)\right),
\end{aligned}
$$

such that the constants $A$ and $A_{l}$ can be expressed as functions of $h(0)$ and $h^{\prime}(0)$,

$$
\begin{aligned}
& A=1-\frac{\left(v-\frac{\lambda}{c}\right) h(0)}{h(0)\left(v-\frac{\lambda}{c}-\frac{\omega\left(0^{-}\right)}{c}\right)+h^{\prime}(0)}, \\
& A_{l}=-\frac{v-\frac{\lambda}{c}}{h(0)\left(v-\frac{\lambda}{c}-\frac{\omega\left(0^{-}\right)}{c}\right)+h^{\prime}(0)},
\end{aligned}
$$

to give the following result.

Proposition 2.1. Consider a compound Poisson surplus process (as defined in (1)) with initial capital $x$, income rate $c$, intensity $\lambda>0$ and exponentially distributed claim sizes with parameter $v>0$. For a given bankruptcy rate function $\omega(x) \geq 0$ for $x<0$, the probability of bankruptcy is given by

$$
\psi(x)=\left\{\begin{array}{cc}
{\left[1-\frac{\left(v-\frac{\lambda}{c}\right) h(0)}{h(0)\left(v-\frac{\lambda}{c}-\frac{\omega\left(0^{-}\right)}{c}\right)+h^{\prime}(0)}\right] e^{-(v-\lambda / c) x,}} & x \geq 0 \\
1-\frac{v-\frac{\lambda}{c}}{h(0)\left(v-\frac{\lambda}{c}-\frac{\omega\left(0^{-}\right)}{c}\right)+h^{\prime}(0)} \cdot h(x), & x<0,
\end{array}\right.
$$

where $h(x)$ is defined as the homogeneous solution to (14).

From the form of $\psi(x)$ in (18), it is obvious that the two branches of the function meet in $x=0$. Also, $A$ and $A_{l}$ remain unchanged if $c \cdot h(x)$ is used instead of $h(x)$ for some constant factor $c \neq 0$, such that it suffices to determine $h(x)$ up to this scaling factor. Note that $h(0), h^{\prime}(0)$ and $\omega\left(0^{-}\right)$will depend on the choice of the bankruptcy rate function, and in the next section some particular choices of such functions will be discussed in more detail.

Remark. The transition from integro-differential equations (IDEs) to ordinary differential equations (ODEs) as performed in this section is not limited to the exponential claim size case. Suppose that the density function 
$f(y)$ of the claim size distribution exists and that it is the solution of the homogeneous ODE

$$
p_{Y}\left(\frac{d}{d x}\right) f(x):=f^{(m)}(x)+d_{m-1} f^{(m-1)}(x)+\cdots+d_{1} f^{\prime}(x)+d_{0} f(x)=0
$$

for constants $d_{j} \in \mathbb{R}$ and $d_{0} \neq 0$ (or, equivalently, the distribution shall have a rational Laplace transform, cf. e.g. Asmussen and Albrecher, 2010). This property is, for example, fulfilled by the rich class of phase-type distributions (which can theoretically be applied to approximate any distribution on the positive half-line arbitrarily well) including hyper-exponential and Erlang distributions. Applying the operator $d^{k} / d x^{k}$ to (11), then returns

$$
\begin{aligned}
0= & \frac{d^{k}}{d x^{k}}\left[c \psi_{u}^{\prime}(x)-\lambda \psi_{u}(x)\right]+\lambda\left(\sum_{i=0}^{k-1} \psi_{u}^{(k-1-i)}(x) f^{(i)}(0)\right. \\
& \left.+\int_{0}^{x} \psi_{u}(y) \frac{d^{k}}{d x^{k}} f(x-y) d y+\int_{-\infty}^{0} \psi_{l}(y) \frac{d^{k}}{d x^{k}} f(x-y) d y\right),
\end{aligned}
$$

and due to (19) one eventually arrives at the ODE

$$
x \geq 0: 0=p_{Y}\left(\frac{d}{d x}\right)\left[c \psi_{u}^{\prime}(x)-\lambda \psi_{u}(x)\right]+\lambda \sum_{k=1}^{m} \sum_{i=0}^{k-1} d_{k} \psi_{u}^{(k-1-i)}(x) f^{(i)}(0)
$$

with $d_{m}=1$. Similarly, the functional $p_{Y}\left(\frac{d}{d x}\right)$ transforms (12) into the ODE

$$
\begin{aligned}
x<0: 0= & p_{Y}\left(\frac{d}{d x}\right)\left[c \psi_{l}^{\prime}(x)-\lambda \psi_{l}(x)\right]-\sum_{k=0}^{m} \sum_{i=0}^{k} d_{k}\left(\begin{array}{c}
k \\
i
\end{array}\right) \omega^{(k-i)}(x) \psi^{(k)}(x) \\
& +\sum_{k=0}^{m} d_{k} \omega^{(k)}(x)+\lambda \sum_{k=1}^{m} \sum_{i=0}^{k-1} d_{k} \psi_{u}^{(k-1-i)}(x) f^{(i)}(0) .
\end{aligned}
$$

Note that the ODE for $x \geq 0$ does not depend on $\omega(\cdot)$ and gives the same dynamics as in the classical case. It remains to solve this new system of higherorder ODEs in order to attain an expression for the probability of bankruptcy. This, however, can be a cumbersome task; one might not be able to identify an analytical solution to the ODE for the lower branch $(x<0)$ with its generally non-constant coefficients. In the case of non-exponential claim size distributions, it might hence be advisable to turn to alternative numerical solution methods (e.g. as the ones described in Sections 2.2 and 3.3). 


\subsection{Examples of bankruptcy rate functions}

2.1.1. Constant bankruptcy rate functions. Let $\omega_{1}(x) \equiv \omega_{c} \cdot \mathbf{1}_{\{x<0\}}$ with $\omega_{c}>0$. This is the simplest choice of bankruptcy rate function and could, for example, be used when the exact deficit level is not transparent in practice (lack of information) or when the surplus is observed only at discrete times. The latter corresponds to the case where the periods between observation times are assumed i.i.d. exponentially distributed with an expected time between observations of $1 / \omega_{c}$ (cf. Albrecher et al., 2011b). The dynamics of $h(x)$ are then given by

$$
0=c h^{\prime \prime}(x)+\left(v c-\left(\lambda+\omega_{c}\right)\right) h^{\prime}(x)-v \omega_{c} h(x), \quad x<0 .
$$

We obtain

$$
h(x)=A_{1} e^{-R x}+B_{1} e^{\rho x},
$$

with $-R<0$ and $\rho>0$ being the two solutions to the characteristic equation

$$
\xi^{2}+\left(v-\frac{\lambda+\omega_{c}}{c}\right) \xi-\frac{v \omega_{c}}{c}=0
$$

The lower boundary condition $\lim _{x \rightarrow-\infty} h(x)=0$ implies $A_{1}=0$, and we choose w.l.o.g. $B_{1}=1$. From the resulting $h(0)=1$ and $h^{\prime}(0)=\rho$, one ultimately finds

$$
A=\frac{\omega_{c}-\rho c}{-c v+\lambda+\omega_{c}-\rho c}=1-\frac{v-\lambda / c}{R},
$$

so that one arrives at the following result.

Proposition 2.2. Consider a compound Poisson surplus process (as defined in (1) with initial capital $x$, income rate $c$, intensity $\lambda>0$ and exponentially distributed claim sizes with parameter $v>0$. For a constant bankruptcy rate function $\omega(x)=\omega_{c}$ for $x<0$, the probability of bankruptcy is given by

$$
\psi(x)= \begin{cases}\frac{R-(\nu-\lambda / c)}{R} e^{-(\nu-\lambda / c) x}, & x \geq 0 \\ 1-\frac{1}{R}(\nu-\lambda / c) e^{\rho x}, & x<0,\end{cases}
$$

where $-R<0$ and $\rho>0$ are solutions of (20).

Note that this result corresponds to the case $\delta=0$ of Formula (2.18) of Albrecher et al. (2011b), where a risk model with random exponential observation times is considered. Also, for $\omega_{c} \rightarrow \infty$, we have $R \rightarrow v$ and $\rho \rightarrow \infty$, so that (21) converges to (16) for $x \geq 0$ and to 1 for $x<0$.

2.1.2. Linear bankruptcy rate functions. Let now $\omega_{2}(x)=-a x \cdot \mathbf{1}_{\{x<0\}}$, for some $a>0$. This case reflects one of the most basic choices of strictly decreasing bankruptcy rate function, which is simple enough to enable an explicit solution 
of the probability of bankruptcy, which can be used to gain insight in parameter sensitivities. In this case, the dynamics of $h(x)$ are given by

$$
0=\operatorname{ch}^{\prime \prime}(x)+(v c-(\lambda-a x)) h^{\prime}(x)+(a+v a x) h(x), \quad x<0 .
$$

Substituting $h(x)=e^{\frac{x(2 \lambda-a x)}{2 c}} \cdot g(x)$ yields a linear ODE for $g(x)$ as

$$
0=c g^{\prime \prime}(x)+(\lambda+c \nu-a x) g^{\prime}(x)+\lambda \nu g(x) .
$$

A second substitution, $z=\frac{(-\lambda-c v+a x)^{2}}{2 a c}$, such that $y(z(x))=g(x)$, produces the Kummer differential equation (also known as Weiler's canonical form),

$$
0=z \frac{d^{2} y}{d z^{2}}+\left(\frac{1}{2}-z\right) \frac{d y}{d z}+\frac{\lambda v}{2 a} y
$$

This equation is well-known to have the general solution (cf. Abramowitz and Stegun, 1972, Section 13)

$$
y(z)=A_{2} \cdot \mathrm{M}\left(-\frac{\lambda \nu}{2 a}, \frac{1}{2}, z\right)+B_{2} \cdot \mathrm{U}\left(-\frac{\lambda \nu}{2 a}, \frac{1}{2}, z\right),
$$

with $A_{2}, B_{2} \in \mathbb{R}, \mathrm{M}(\alpha, \beta, z)=1+\sum_{k=1}^{\infty} \frac{(\alpha)_{k}}{(\beta)_{k}} \frac{z_{k}^{k}}{k !}$ being the Kummer series (also known as confluent hypergeometric series of the first kind, $\left.{ }_{1} F_{1}(\alpha, \beta, z)\right)$ for $\beta \notin$ $\mathbb{Z}_{0}^{-}$, and $(\alpha)_{k}=\alpha(\alpha+1) \ldots(\alpha+k-1),(\alpha)_{0}=1$, and one defines

$$
\begin{aligned}
\frac{\sin \pi \beta}{\beta} U(\alpha, \beta, z)= & \frac{1}{\Gamma(1+\alpha-\beta) \Gamma(\beta)} M(\alpha, \beta, z) \\
& -\frac{z^{1-\beta}}{\Gamma(\alpha) \Gamma(2-\beta)} M(1+\alpha-\beta, 2-\beta, z) .
\end{aligned}
$$

This leads to the general solution of (22),

$$
\begin{aligned}
h(x)= & e^{\frac{x(2 \lambda-a x)}{2 c}} \cdot\left[A_{2} \cdot \mathrm{M}\left(-\frac{\lambda \nu}{2 a}, \frac{1}{2}, \frac{(-\lambda-c \nu+a x)^{2}}{2 a c}\right)\right. \\
& \left.+B_{2} \cdot \mathrm{U}\left(-\frac{\lambda \nu}{2 a}, \frac{1}{2}, \frac{(-\lambda-c \nu+a x)^{2}}{2 a c}\right)\right] .
\end{aligned}
$$

For $z \rightarrow \infty$, it is well-known that $M(\alpha, \beta, z)=\frac{\Gamma(\beta)}{\Gamma(\alpha)} z^{z} z^{\alpha-\beta}(1+O(1 /|z|))$ and $U(\alpha, \beta, z)=z^{-\alpha}(1+O(1 /|z|))$. Hence, for $x \rightarrow-\infty$, the two homogeneous solutions have the asymptotic behavior

$$
\frac{\Gamma(1 / 2)}{\Gamma(-\lambda v /(2 a))} e^{-v x}\left(\frac{-\lambda-c v+a x}{\sqrt{2 a c}}\right)^{-\lambda v / a-1} O\left(1+\frac{1}{|x|}\right)
$$


and

$$
e^{\frac{x(2 \lambda-a x)}{2 c}}\left(\frac{-\lambda-c v+a x}{\sqrt{2 a c}}\right)^{-\lambda v / a+2} O\left(1+\frac{1}{|x|}\right)
$$

respectively. For $x \rightarrow-\infty$, (24) is unbounded, while (25) tends to 0 . The lower boundary condition $\lim _{x \rightarrow-\infty} h(x)=0$ thus implies $A_{2}=0$, and w.l.o.g. $B_{2}=1$. It is concluded that

$$
h(x)=e^{\frac{x(2 \lambda-a x)}{2 c}} \cdot \mathrm{U}\left(-\frac{\lambda \nu}{2 a}, \frac{1}{2}, \frac{(-\lambda-c \nu+a x)^{2}}{2 a c}\right)
$$

and it follows that

$$
\begin{aligned}
h(0)= & \mathrm{U}\left(-\frac{\lambda \nu}{2 a}, \frac{1}{2}, \frac{(-\lambda-c \nu)^{2}}{2 a c}\right), \\
h^{\prime}(0)= & \frac{\lambda}{c} \mathrm{U}\left(-\frac{\lambda \nu}{2 a}, \frac{1}{2}, \frac{(-\lambda-c v)^{2}}{2 a c}\right) \\
& +\frac{\lambda \nu(\lambda+c \nu)}{2 a c} \mathrm{U}\left(-\frac{\lambda v}{2 a}+1, \frac{3}{2}, \frac{(-\lambda-c \nu)^{2}}{2 a c}\right),
\end{aligned}
$$

leading to the following result.

Proposition 2.3. Consider a compound Poisson surplus process (as defined in (1) with initial capital $x$, income rate $c$, intensity $\lambda>0$ and exponentially distributed claim sizes with parameter $v>0$. For a linear bankruptcy rate function $\omega(x)=-$ ax for $x<0$, the probability of bankruptcy is given by



where $U(\cdot)$ is the Kummer series as defined in (23).

While the above formulas look quite complex, it is straightforward to evaluate them for specific values of $a, c, \lambda$ and $v$ to attain an explicit representation for the probability of bankruptcy. 
2.1.3. Exponential bankruptcy rate functions. Let now $\omega_{3}(x)=e^{-a x} \cdot \mathbf{1}_{\{x<0\}}$, for some $a>0$. Such a function choice could be better suited than its linear counterpart to approximate targeted bankruptcy rates by a simple one-parameter function, when bankruptcy rates for lower absolute levels of negative surplus are expected to be somewhat flat while the exponential shape will ensure higher rates for higher deficit levels. The differential equation for $h(x)$ is given by

$$
0=c h^{\prime \prime}(x)+\left(v c-\left(\lambda+e^{-a x}\right)\right) h^{\prime}(x)-e^{-a x}(v-a) h(x), \quad x<0 .
$$

Techniques similar to the ones applied in the linear case lead to a solution. The substitutions $h(x)=e^{-\frac{e^{-a x}}{a c}} \cdot g(x)$ and, subsequently, $z=\frac{1}{a c} e^{-a x}$, with $y(z(x))=g(x)$, lead again to a Kummer differential equation, and this time it is of the form

$$
0=z \frac{d^{2} y}{d z^{2}}+\left(\frac{a c+\lambda-v c}{a c}-z\right) \frac{d y}{d z}-\frac{\lambda}{a c} y .
$$

From the general solution to the above, one finds

$$
\begin{aligned}
h(x)= & e^{-\frac{e^{-a x}}{a c}} \cdot\left[A_{3} \cdot \mathrm{M}\left(\frac{\lambda}{a c}, \frac{\lambda-v c}{a c}+1, \frac{e^{-a x}}{a c}\right)\right. \\
& \left.+B_{3} \mathrm{U}\left(\frac{\lambda}{a c}, \frac{\lambda-v c}{a c}+1, \frac{e^{-a x}}{a c}\right)\right]
\end{aligned}
$$

for constants $A_{3}, B_{3} \in \mathbb{R}$.

Remark. When choosing the more general bankruptcy rate function $\widetilde{\omega_{3}}(x)=$ $b \cdot e^{-a x} \cdot \mathbf{1}_{\{x<0\}}$, one would simply have to replace all terms $\frac{e^{-a x}}{a c}$ in (27) by $\frac{b e^{-a x}}{a c}$ to attain the general solution $h(x)$, and we restrict computations in this section to the case $b=1$ to keep notation compact. In this context, $\widetilde{\omega_{3}}(x)$ can be interpreted as the failure rate of a Gompertz distribution.

Examining the asymptotic behavior for $x \rightarrow-\infty$, the two homogeneous solutions in (27) behave as

$$
\frac{\Gamma\left(\frac{\lambda-v c}{a c}+1\right)}{\Gamma\left(\frac{\lambda}{a c}\right)}\left(\frac{e^{-a x}}{a c}\right)^{\frac{v}{a}-1} O\left(1+\frac{1}{|x|}\right)
$$

and

$$
e^{-\frac{e^{-a x}}{a c}}\left(\frac{e^{-a x}}{a c}\right)^{-\frac{\lambda}{a c}} O\left(1+\frac{1}{|x|}\right),
$$

respectively. Let us assume that $v>a$ in the sequel (by the lack-of-memory property of the exponential distribution, the first jump to a negative surplus level always leads to an exponentially distributed deficit and one sees that $\mathbb{E}\left[\omega_{3}(Y)\right]<$ $\infty$ only if $v>a$ ). Then the first homogeneous solution in (27) is unbounded for $x \rightarrow-\infty$ (cf. (28)), while the latter tends to 0 . Again it is required that 
$\lim _{x \rightarrow-\infty} h(x)=0$, such that $A_{3}=0$ and w.l.o.g. $B_{3}=1$. It follows that

$$
h(x)=e^{-\frac{e^{-a x}}{a c}} \cdot \mathrm{U}\left(\frac{\lambda}{a c}, \frac{\lambda-v c}{a c}+1, \frac{e^{-a x}}{a c}\right),
$$

and in particular

$$
\begin{aligned}
h(0)= & e^{-\frac{1}{a c}} \cdot \mathrm{U}\left(\frac{\lambda}{a c}, \frac{\lambda-v c}{a c}+1, \frac{1}{a c}\right), \\
h^{\prime}(0)= & e^{-\frac{1}{a c}} \cdot\left[\frac{1}{c} \mathrm{U}\left(\frac{\lambda}{a c}, \frac{\lambda-v c}{a c}+1, \frac{1}{a c}\right)\right. \\
& \left.+\frac{\lambda}{a c^{2}} \mathrm{U}\left(\frac{\lambda}{a c}+1, \frac{\lambda-v c}{a c}+2, \frac{1}{a c}\right)\right],
\end{aligned}
$$

so that the following result is obtained.

Proposition 2.4. Consider a compound Poisson surplus process ((as defined in (1)) with initial capital $x$, income rate $c$, intensity $\lambda>0$ and exponentially distributed claim sizes with parameter $v>0$. For an exponential bankruptcy rate function $\omega(x)=\exp (-a x)$ for $x<0$, the probability of bankruptcy is given by

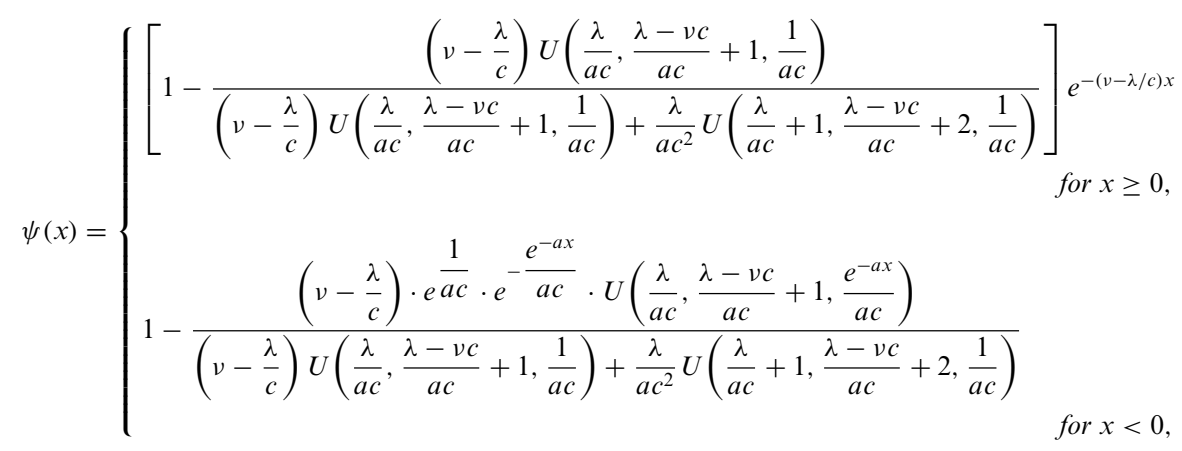

where $U(\cdot)$ is the Kummer series as defined in (23).

From the examples in this section it becomes clear that finding an explicit expression of the probability of bankruptcy can be cumbersome. Computationally efficient numerical alternatives include simulation (cf. Section 3.3) and piecewise constant approximations of the bankruptcy rate functions as discussed in the following.

\subsection{Piecewise constant bankruptcy rate functions}

Piecewise constant functions are of particular interest, as they can approximate arbitrarily closely any given function from below and above. Furthermore, in 




FIGURE 1: Upper and lower approximation of a bankruptcy rate function $\omega_{0}\left(C_{t}\right)$ on a grid $-\infty=x_{0}, x_{1}, \ldots, x_{n}=0$.

some situations it may be sufficient to assign bankruptcy rates to certain ranges of negative surplus. The choice of such rates and their ranges may depend on in-place contingent capital arrangements, local insurance regulation, etc.

Define a grid on the negative half-line by choosing distinct values $x_{i}, 0 \leq i \leq$ $n$, such that $-\infty=x_{0}<x_{1}<\ldots<x_{n-1}<x_{n}=0$, and set

$$
\omega(x)=\omega_{k} \text { for } x_{k-1}<x \leq x_{k},
$$

with the typical requirement $\omega_{1}>\omega_{2}>\cdots>\omega_{n} \geq 0$. Suppose, one would like to approximate some bankruptcy rate function $\omega_{0}(x)$. As in Albrecher et al. (2011), one can produce a lower piecewise constant approximation by choosing $\omega_{k}=\omega_{0}\left(x_{k}\right)$. Similarly, $\omega_{0}(x)$ can be approximated from above by choosing $\omega_{k}=\omega_{0}\left(x_{k-1}\right)$ (cf. Figure 1). These lower and upper approximations of $\omega_{0}(x)$ will subsequently provide lower and upper bounds for the probability of bankruptcy, respectively.

Under (34), (14) can be re-written as $n$ differential equations, describing the local dynamics depending on the value of $x$,

$$
x_{k-1}<x<x_{k}: 0=c \psi_{k}^{\prime \prime}(x)+\left(v c-\left(\lambda+\omega_{k}\right)\right) \psi_{k}^{\prime}(x)-v \omega_{k} \psi_{k}(x)+v \omega_{k} .
$$

Each differential equation has now constant coefficients so that we solve

$$
\psi_{k}(x)=A_{k} e^{-r_{k} x}+B_{k} e^{\rho_{k} x}+1, x_{k-1}<x<x_{k},
$$

where $-r_{k}<0$ and $\rho_{k}>0$ are the solutions of the characteristic equation

$$
\xi^{2}+\left(v-\frac{\lambda+\omega_{k}}{c}\right) \xi-\frac{v \omega_{k}}{c}=0
$$


$A_{k}$ and $B_{k}$ are constants to be determined. Note that this form of the solution even applies if $\omega_{k}=0$ as the constant term is then simply $B_{k}+1$.

Conditions on the coefficients. As in the previous section, we have the outer boundary conditions $\lim _{x \rightarrow \infty} \psi_{u}(x)=0$ and $\lim _{x \rightarrow-\infty} \psi_{1}(x)=1$, which imply that $B=0$ and $A_{1}=0$.

Imposing continuity at the $x_{k}$ 's yields the conditions

$$
A_{k} e^{-r_{k} x_{k}}+B_{k} e^{\rho_{k} x_{k}}=A_{k+1} e^{-r_{k+1} x_{k}}+B_{k+1} e^{\rho_{k+1} x_{k}},
$$

for $1 \leq k \leq n-1$, and

$$
A_{n}+B_{n}+1=A \text {. }
$$

Substituting the general form (35) of $\psi_{k}(x)$ into (12) produces $n$ more conditions. For $x_{k-1}<x<x_{k}$ and $1 \leq k \leq n$, we find

$$
\begin{aligned}
0= & c\left(-r_{k} A_{k} e^{-r_{k} x}+\rho_{k} B_{k} e^{\rho_{k} x}\right)-\left(\lambda+\omega_{k}\right)\left(A_{k} e^{-r_{k} x}+B_{k} e^{\rho_{k} x}+1\right) \\
& +\omega_{k}+\lambda \int_{0}^{x-x_{k-1}}\left(A_{k} e^{-r_{k}(x-y)}+B_{k} e^{\rho_{k}(x-y)}+1\right) v e^{-v y} d y \\
& +\lambda \sum_{j=1}^{k-1} \int_{x-x_{k-j}}^{x-x_{k-j-1}}\left(A_{k-j} e^{-r_{k-j}(x-y)}+B_{k-j} e^{\rho_{k-j}(x-y)}+1\right) v e^{-v y} d y .
\end{aligned}
$$

The properties

$$
-c r_{k}-\left(\lambda+\omega_{k}\right)+\frac{\lambda v}{v-r_{k}}=0, \quad c \rho_{k}-\left(\lambda+\omega_{k}\right)+\frac{\lambda v}{v+\rho_{k}}=0
$$

can be used to rewrite the first two terms of (38), and evaluating the integrals leads to

$$
\begin{aligned}
0= & \lambda v e^{-v x}\left[-\frac{A_{k}}{v-r_{k}} e^{x_{k-1}\left(v-r_{k}\right)}-\frac{B_{k}}{v+\rho_{k}} e^{x_{k-1}\left(v+\rho_{k}\right)}-\frac{1}{v} e^{x_{k-1} v}\right. \\
& +\sum_{j=2}^{k-1}\left(\frac{A_{j}}{v-r_{j}}\left(-e^{x_{j-1}\left(v-r_{j}\right)}+e^{x_{j}\left(v-r_{j}\right)}\right)+\frac{B_{j}}{v+\rho_{j}}\left(-e^{x_{j-1}\left(v+\rho_{j}\right)}+e^{x_{j}\left(v+\rho_{j}\right)}\right)\right. \\
& \left.\left.+\frac{1}{v}\left(-e^{x_{j-1} v}+e^{\left.x_{j} v\right)}\right)\right)+\frac{B_{1}}{v+\rho} e^{x_{1}(v+\rho)}+\frac{1}{v} e^{x_{1} v}\right], \quad(2 \leq k \leq n)
\end{aligned}
$$


Similarly, we can rearrange the IDE as in (11) for $x \geq 0$ and with $-r=$ $-\left(v-\frac{\lambda}{c}\right)$ to yield

$$
\begin{aligned}
0= & \lambda v e^{-v x}\left[-\frac{A}{v-r}+\frac{A_{n}}{v-r_{n}}\left(1-e^{x_{n-1}\left(v-r_{n}\right)}\right)\right. \\
& +\frac{B_{n}}{v+\rho_{n}}\left(1-e^{x_{n-1}\left(v+\rho_{n}\right)}\right)+\frac{1}{v}\left(1-e^{x_{n-1} v}\right) \\
& +\sum_{j=2}^{k-1}\left(\frac{A_{j}}{v-r_{j}}\left(-e^{x_{j-1}\left(v-r_{j}\right)}+e^{x_{j}\left(v-r_{j}\right)}\right)+\frac{B_{j}}{v+\rho_{j}}\left(-e^{x_{j-1}\left(v+\rho_{j}\right)}+e^{x_{j}\left(v+\rho_{j}\right)}\right)\right. \\
& \left.\left.+\frac{1}{v}\left(-e^{x_{j-1} v}+e^{x_{j} v}\right)\right)+\frac{B_{1}}{v+\rho} e^{x_{1}(v+\rho)}+\frac{1}{v} e^{x_{1} v}\right] .
\end{aligned}
$$

Noting that $\lambda \nu e^{-v x}>0$, one can simplify conditions (39) and (40) to give, for $1 \leq k \leq n-1$,

$$
A_{k} \frac{e^{-r_{k} x_{k}}}{v-r_{k}}+B_{k} \frac{e^{\rho_{k} x_{k}}}{v+\rho_{k}}-A_{k+1} \frac{e^{-r_{k+1} x_{k}}}{v-r_{k+1}}-B_{k+1} \frac{e^{\rho_{k+1} x_{k}}}{v+\rho_{k+1}}=0
$$

and

$$
A_{n} \frac{1}{v-r_{n}}+B_{n} \frac{1}{v+\rho_{n}}-A \frac{1}{v-r}=-\frac{1}{v} .
$$

As it is our aim to solve for the parameters $B_{1}, A_{k}, B_{k}$, A, with $2 \leq k \leq n$, we summarize all attained conditions, (36), (37), (41) and (42), as a linear equation system of the form

$$
U \cdot z=v .
$$

Here

$$
z^{\prime}=\left[B_{1}, A_{2}, B_{2}, \ldots, A_{n}, B_{n}, A\right],
$$

the matrix $U$ is of dimension $2 n \times 2 n$, scarcely populated around the main diagonal and given by

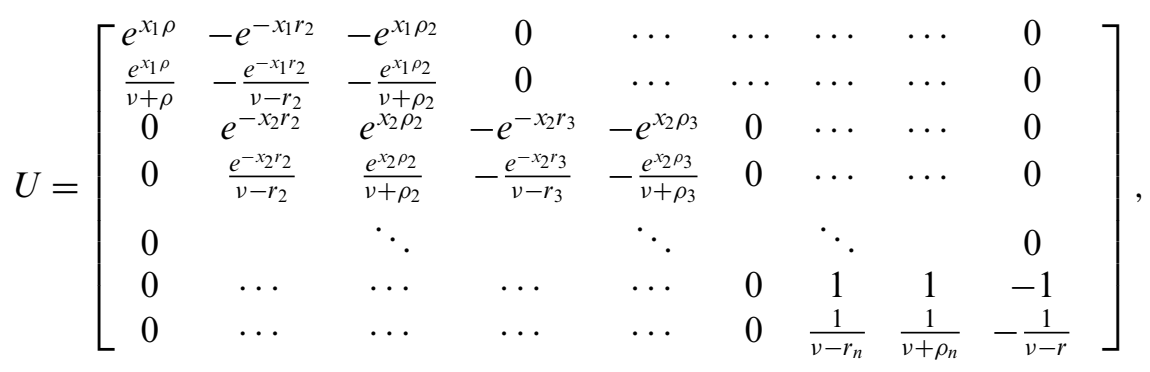

and

$$
v^{\prime}=\left[0,0,0, \ldots,-1,-\frac{1}{v}\right]
$$


For example, one can now write the probability of bankruptcy for $x \geq 0$ as

$$
\psi(x)=\left(U^{-1} v\right)_{2 n} e^{-\left(v-\frac{\lambda}{c}\right) x},
$$

where the index indicates the $2 n$th component of the vector.

\section{ILLUSTRATIVE COMPUTATIONS OF THE PROBABILITY OF BANKRUPTCY}

We now illustrate the evaluation of $\psi(x)$ for linear and exponential bankruptcy rate functions $\omega_{2}(x)=-a x$ and $\omega_{3}(x)=e^{-a x}$, respectively, and for several parameter values $a>0$. The results will be compared with the values of the classical ruin probability $\psi_{\text {ruin }}(x)$. The first set of plots is obtained by evaluating the explicit formulas for the probability of bankruptcy as in (26) and (33). This is then compared with the corresponding results based on piecewise constant approximation of the respective bankruptcy rate functions as described in Section 2.2. The probability of bankruptcy for given initial capital is then attained through solving the linear equation system (43). Finally, as an additional computational alternative, a Monte Carlo simulation algorithm will be implemented. All illustrations will be based on the following parameters. Let $\lambda=5,000, v=1, c=6,000$. This could correspond to a collective risk model approximating a portfolio of 100,000 independent policies, each having a claim probability of $q=5 \%$ per time unit, an expected claim size given its occurrence of $\mathbb{E}[Y]=\frac{1}{v}=1$, and a premium loading of $\theta=20 \%$, i.e. $c=(1+\theta) \mathbb{E}\left[\sum_{i=1}^{N(1)} Y_{i}\right]=1.2 \times 5,000=6,000$. In the linear case, we depict $\psi(x)$ for $a_{\text {lin }} \in\{1,10,100\}$ and in the exponential case $a_{\text {exp }} \in\{0.1,0.2,1\}$. The results are plotted in comparison to the classical ruin probability in order to illustrate the effect of introducing the bankruptcy concept.

\subsection{Plotting the explicit solution}

Figures 2 and 3 were produced by evaluating the explicit expressions in (26) and (33). As expected, the functions show a smooth shape and, compared to the classical ruin case, are pulled more and more to the left as $a$ is increased.

\subsection{Piecewise constant approximations of $\omega(x)$}

3.2.1. Methodology. When numerically solving the linear equation system (43), we observe that the matrix is scarcely populated around the diagonal and we find the Gauss-Seidel method to produce satisfactory results (with noniterative methods we were facing numerical stability issues due to the alternately very large and small coefficients in $U$ for some parameter sets). The 


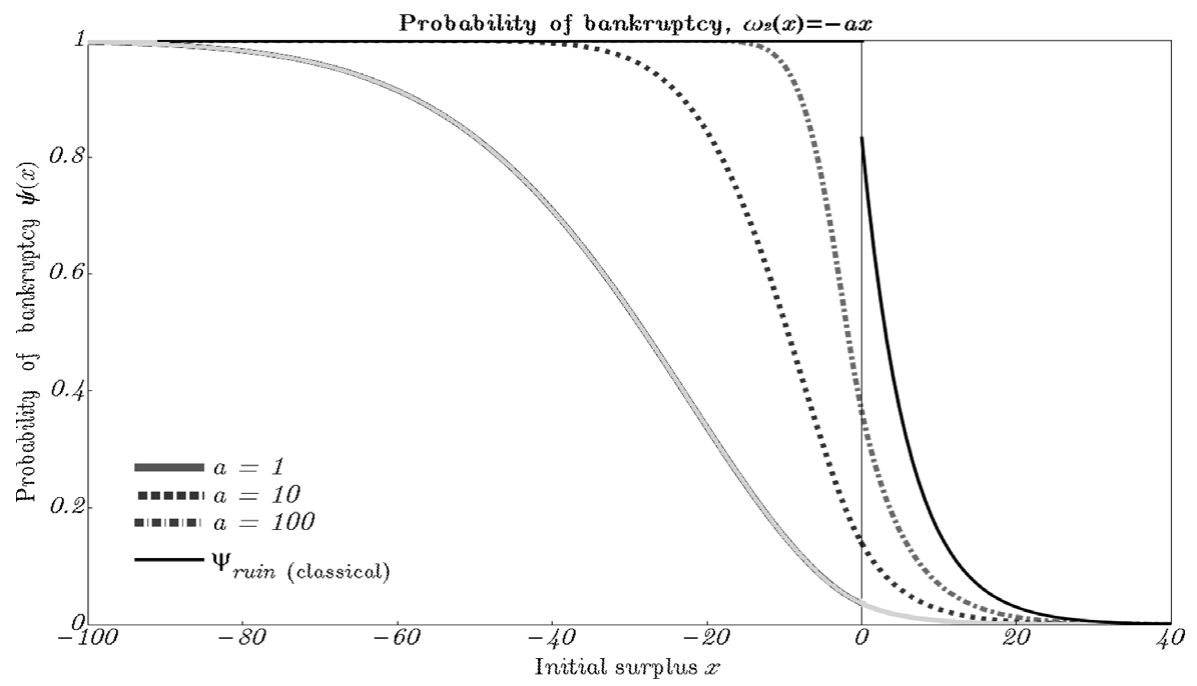

FIGURE 2: Probabilities of bankruptcy for linear $\omega_{2}(x)$ and various parameter levels $a$.

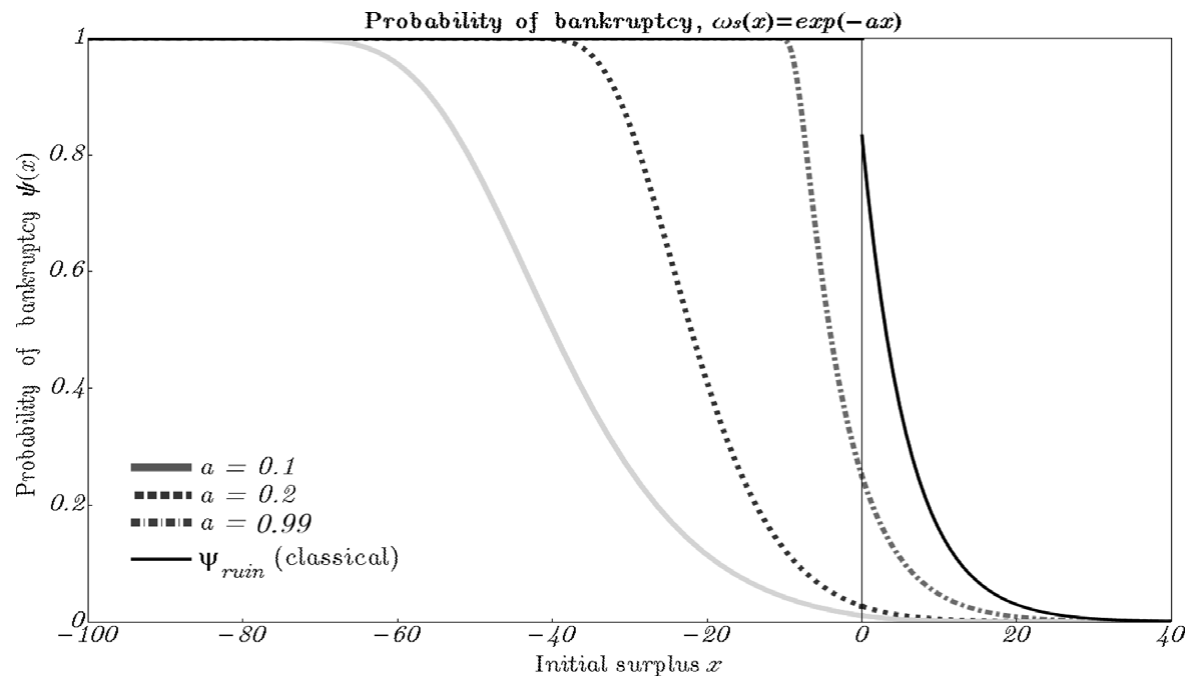

FIGURE 3: Probabilities of bankruptcy for exponential $\omega_{3}(x)$ and various parameter levels $a$.

Gauss-Seidel algorithm applies the iteration law

$$
z_{k}^{(m+1)}=\frac{1}{u_{k k}}\left(v_{k}-\sum_{i=1}^{k-1} u_{k i} z_{i}^{(m+1)}-\sum_{i=k+1}^{n} u_{k i} z_{i}^{(m)}\right),
$$



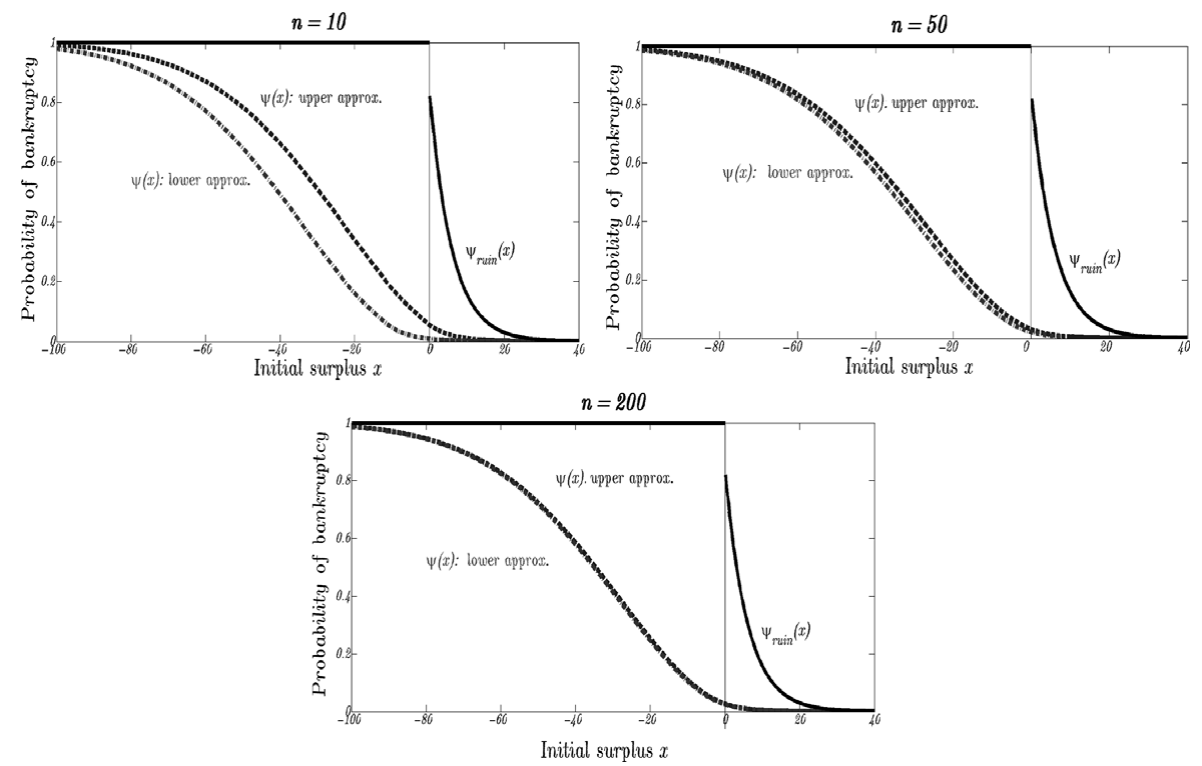

FIGURE 4: Upper and lower approximations of the probability of bankruptcy for $\omega_{2}(x)$ and given $x_{1}=-100$ depending on the grid width $n$.

where $z^{(m)}$ is the step- $m$ approximation of the solution vector $z$, with $z^{(m)} \rightarrow z$ for $m \rightarrow \infty$ such that $U \cdot z=v$. If one sets $U=L+D+R$, where $D$ is a diagonal matrix, and $L$ and $R$ are strictly lower and upper diagonal matrices, it can be shown that the method converges as the spectral radius of $-(D+L)^{-1} R$ is smaller than 1 . The algorithm is terminated with $z^{(m+1)}$ as the approximation of the solution $z$ once $\left\|z^{(m+1)}-z^{(m)}\right\|_{\max }<\varepsilon$ and for the present purposes we found $\varepsilon=10^{-10}$ to give satisfactory results. The grid $x_{1}, x_{2}, \ldots, x_{n}$ is set in an equidistant way such that the quality of the approximation is mainly driven by the choice of $x_{1}$ and the number $n$ of grid points.

\subsubsection{Results.}

Linear bankruptcy rate functions. Convergence of the upper and lower approximations is illustrated in Figure 4, where we use $\omega_{2}(x)=-a x, a=1$ and $x_{1}=-100$. The dashed line depicts the probability of bankruptcy for the upper approximation of the bankruptcy rate function, i.e. the case where $\omega_{2}(x)=\omega_{2}\left(x_{i}\right)$ for $x_{i} \leq x<x_{i+1}$, and the dash-dotted line gives the lower approximation with $\omega_{2}(x)=\omega_{2}\left(x_{i}\right)$ for $x_{i-1} \leq x<x_{i}$. The solid line shows the classical ruin probability as a reference point. As the width of the grid becomes more dense, the upper and lower bounds converge as expected (note that the discontinuities of $\psi_{1}^{\prime}(x)$ at the discontinuity points of the bounding step-function for $\omega_{2}$ are of negligible magnitude here). Table 1 depicts the influence of $x_{1}$ on 
TABLE 1

THE IMPACT OF $x_{1}$ ON THE UPPER AND LOWER APPROXIMATIONS OF THE $A$ COEFFICIENT.

\begin{tabular}{lcccccccc}
\hline \hline$x_{1}$ & -10 & -20 & -30 & -40 & -50 & -60 & -70 & -80 \\
$n$ & 20 & 40 & 60 & 80 & 100 & 120 & 140 & 160 \\
\hline $\begin{array}{l}\text { Upper } \\
\quad \text { approx. } A\end{array}$ & 0.1026 & 0.0371 & 0.0279 & 0.0268 & 0.0267 & 0.0267 & 0.0267 & 0.0267 \\
$\begin{array}{l}\text { Lower } \\
\text { approx. } A\end{array}$ & 0.0212 & 0.0241 & 0.0245 & 0.0245 & 0.0245 & 0.0245 & 0.0245 & 0.0245 \\
\hline \hline
\end{tabular}

TABLE 2

APPROXIMATION OF $A$ IN THE PROBABILITY OF BANKRUPTCY (CORRESPONDING TO $x>0$ ), FOR LINEAR $\omega_{2}(x)$ AND VARIOUS VALUES $a$.

\begin{tabular}{lcccccc}
\hline \hline$a$ & 1 & 5 & 10 & 20 & 50 & 100 \\
\hline Upper approx. $A$ & 0.0261 & 0.0911 & 0.1401 & 0.2015 & 0.2960 & 0.3719 \\
Lower approx. $A$ & 0.0250 & 0.0874 & 0.1344 & 0.1933 & 0.2839 & 0.3566 \\
\hline \hline
\end{tabular}

the upper and lower approximation of the $A$ coefficient in the probability of bankruptcy $\psi_{1}(x)=A e^{-\left(v-\frac{\lambda}{c}\right) x}, x>0$.

Note that for the chosen parameters, moving lower from $x_{1}=-40$ does not greatly affect the upper and lower approximations.

In a next step, we illustrate the impact of the bankruptcy rate function $\omega_{2}(x)=-a x$ for various parameter choices $a>0$. Table 2 shows how the coefficient $A$ changes depending on the choice of $a$. As $a$ increases, the coefficient $A$ grows toward $\frac{\lambda}{v c}$ which is the corresponding coefficient in the classical ruin case. The corresponding probabilities of bankruptcy depending on the initial surplus level $x$ are depicted in Figure 5.

Exponential bankruptcy rate function. We can now perform the same analysis for exponential bankruptcy rate functions $\omega_{3}(x)=e^{-a x}$. We again choose $x_{1}=-100$ and $n=200$. In Table 3, we find that for given $a$, the probability of bankruptcy converges to the classical ruin probability faster than for linear bankruptcy rate functions, which is in line with expectations. Hence, we regard a lower parameter range $a$ in comparison to the discussion of the linear case. Note that already for $a=2$, the approximating coefficients $A$ are closer to the corresponding coefficient $\frac{\lambda}{v c}=\frac{5}{6}$ of the classical ruin case, than the linear case with parameter $a=100$. The corresponding probability of bankruptcy functions is depicted in Figure 6, and shows similar shapes to the linear case.

\subsection{Monte Carlo simulation}

Monte Carlo simulation is another alternative to obtain numerical estimates of the bankruptcy probability. 


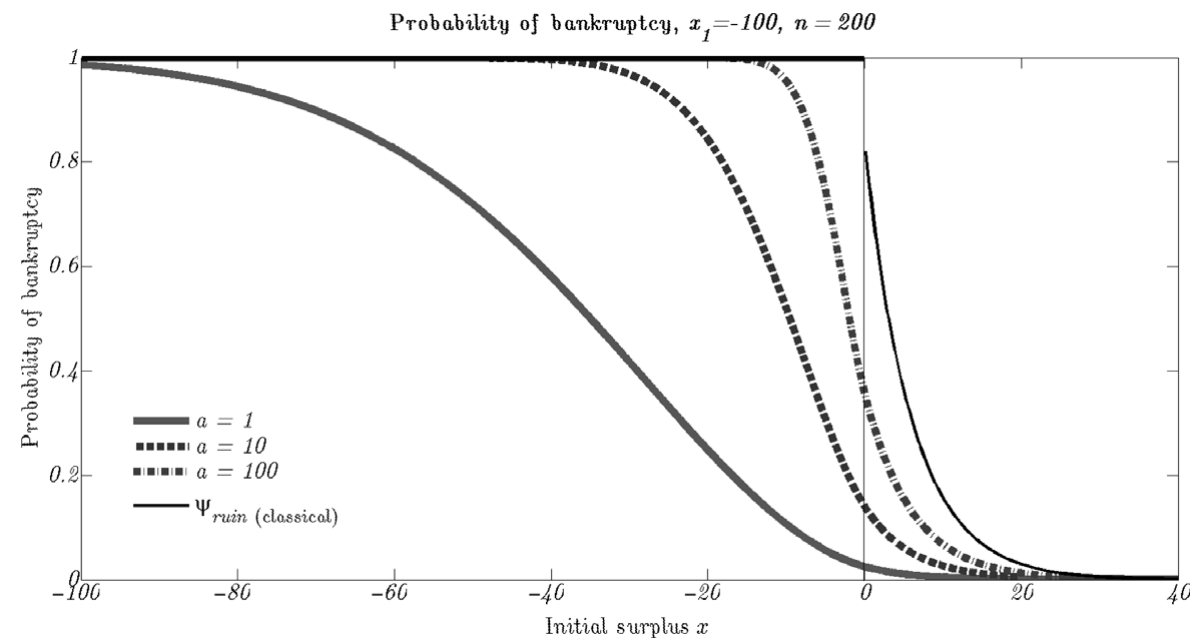

FIGURE 5: Probabilities of bankruptcy for linear $\omega_{2}(x)$ and various parameter levels $a$.

TABLE 3

COEFFICIENTS $A$ IN THE PROBABILITY OF BANKRUPTCY (CORRESPONDING TO $x>0$ ), FOR EXPONENTIAL $\omega_{3}(x)$ AND VARIOUS VALUES $a$.

\begin{tabular}{lcccccc}
\hline \hline$a$ & 0.1 & 0.2 & 0.5 & 1 & 2 & 5 \\
\hline Upper approx. $A$ & 0.0111 & 0.0282 & 0.1172 & 0.2578 & 0.4285 & 0.6220 \\
Lower approx. $A$ & 0.0106 & 0.0263 & 0.1125 & 0.2474 & 0.4110 & 0.5928 \\
\hline \hline
\end{tabular}

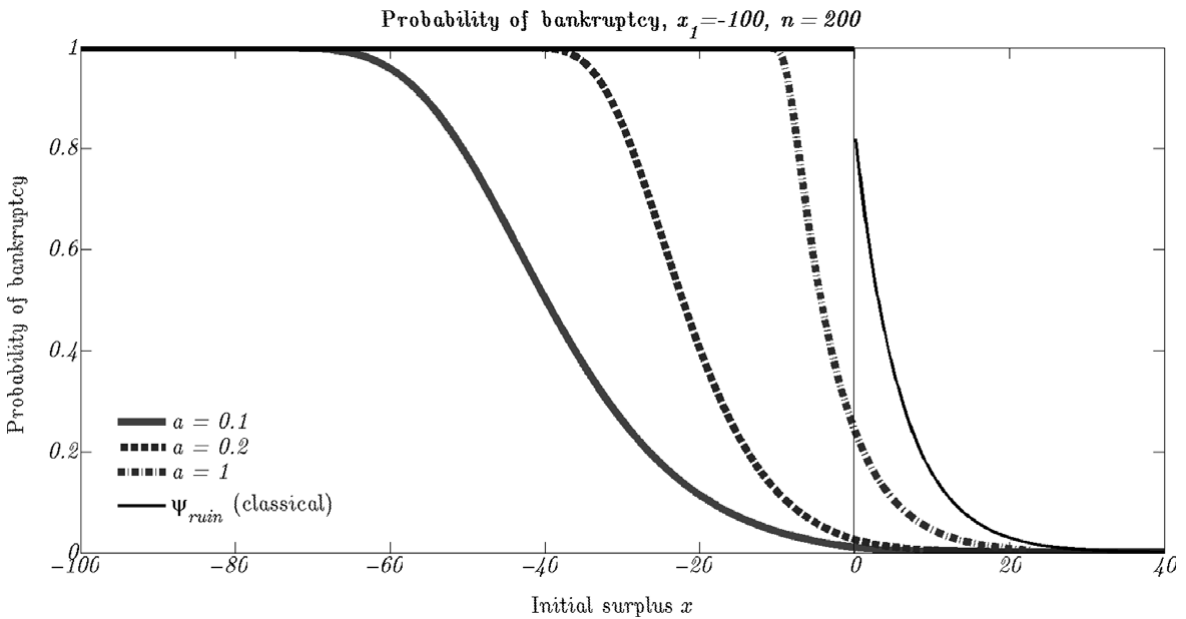

FIGURE 6: Probabilities of bankruptcy for exponential $\omega_{3}(x)$ and various parameter levels $a$. 
3.3.1. Methodology. Note that a crude Monte Carlo approach simulating claims and bankruptcy events for negative surplus levels will be relatively inefficient. However, two observations enable to increase simulation speed significantly.

First, for positive initial surplus levels $x_{+}>0$ we note that, as bankruptcy implies ruin in the classical sense, $\psi\left(x_{+}\right)=\psi_{\text {ruin }}\left(x_{+}\right) \mathbb{P}\left[\tau<\infty \mid \tau_{\text {ruin }}<\infty, C_{0}=\right.$ $\left.x_{+}\right]$. In particular, with exponential claim sizes $Y_{i} \sim \operatorname{Exp}(v)$, it follows that

$$
\psi\left(x_{+}\right)=\frac{\lambda}{v c} e^{-(\nu-\lambda / c) x_{+}} \mathbb{E}[\psi(-D)]
$$

where $D \sim \operatorname{Exp}(v)$. This is due to ruin always occurring at claim times, and the ruin deficit $D$ being again $\operatorname{Exp}(v)$-distributed by the lack-of-memory property of the exponential distribution. Hence, the problem of simulating the probability of bankruptcy for positive initial surplus can be translated into generating sample paths of exponentially distributed negative initial surplus. In this way one avoids generating paths that never become negative.

Second, the computation of $\mathbb{E}[\psi(-D)]$ can be improved compared to a crude Monte Carlo case. It holds for any surplus level $x \in \mathbb{R}$ that

$$
\psi(x)=1-\mathbb{E}\left[e^{-\int_{0}^{\infty} \omega\left(C_{t}\right) \mathbb{1}_{\left\{C_{t}<0\right\}} d t} \mid C_{0}=x\right],
$$

as bankruptcy can only be avoided if there is no event of the Poisson process with level-dependent intensity $\omega(\cdot)$ during the time the process spends on the negative half-line. The above expectation can then be computed by conditioning on the simulated sample path. Concretely, conditioning on the jump times $T_{i}$ and jump sizes $\Theta_{i}$, with

$$
\begin{aligned}
\Psi(\omega, u) \mid\left(T_{1}, \Theta_{1}\right),\left(T_{2}, \Theta_{2}\right) \ldots & =-\int_{0}^{\infty} \omega\left(C_{t}\right) \cdot \mathbb{1}_{\left\{C_{t}<0\right\}} d t \\
& =-\sum_{i=0}^{\infty} \mathbb{1}_{\left\{C_{\left.T_{i}<0\right\}}\right.} \int_{T_{i}}^{\min \left(T_{i+1}, T_{i}-C_{T_{i}} / c\right)} \omega\left(C_{S}\right) d s
\end{aligned}
$$

with $T_{0}=0$, we can write

$$
\psi(\omega, u)=\mathbb{E}_{\left(T_{1}, \Theta_{1}\right),\left(T_{2}, \Theta_{2}\right) \ldots}\left[1-e^{\Psi(\omega, u) \mid\left(T_{1}, \Theta_{1}\right),\left(T_{2}, \Theta_{2}\right) \ldots}\right]
$$






FIGURE 7: Computation of $\Psi(-x, u)$ conditional on a realized sample path.

In particular, for the two choices $\omega_{2}(x)=-a x$ and $\omega_{3}(x)=e^{-a x}, a>0$, (46) reads

$$
\begin{aligned}
& \Psi\left(\omega_{2}, u\right) \mid\left(T_{1}, \Theta_{1}\right),\left(T_{2}, \Theta_{2}\right) \ldots \\
& =\sum_{i=0}^{\infty} \mathbb{1}_{\left\{C_{\left.T_{i}<0\right\}}\right\}} \cdot a\left[\left(C_{T_{i}}-c T_{i}\right)\left(\min \left[T_{i+1}-T_{i},-\frac{C_{T_{i}}}{c}\right]\right)\right. \\
& \left.\quad+\frac{c}{2}\left(\left(\min \left[T_{i+1}, T_{i}-\frac{C_{T_{i}}}{c}\right]\right)^{2}-T_{i}^{2}\right)\right],
\end{aligned}
$$

and

$$
\begin{aligned}
\Psi\left(\omega_{3}, u\right) \mid\left(T_{1}, \Theta_{1}\right),\left(T_{2}, \Theta_{2}\right) \ldots= & \sum_{i=0}^{\infty} \mathbb{1}_{\left\{C_{\left.T_{i}<0\right\}}\right.} \cdot \frac{1}{a c} e^{-a\left(C_{T_{i}}-c T_{i}\right)} \\
& \cdot\left[e^{-a c\left(\min \left(T_{i+1}, T_{i}-C_{T_{i}} / c\right)\right)}-e^{-a c T_{i}}\right] .
\end{aligned}
$$

Figure 7 depicts a particular path, and the shaded area refers to $\Psi_{2}(-x, u) \mid\left(T_{1}, \Theta_{1}\right),\left(T_{2}, \Theta_{2}\right) \ldots$ as in (47).

In the following simulations, $n$ surplus paths are generated and for the $k$ th such path, the function $\Psi(\omega, u)_{k} \mid\left(T_{1}, \Theta_{1}\right),\left(T_{2}, \Theta_{2}\right) \ldots$ is computed as per (47) and (48). The estimator of the bankruptcy probability is then given by

$$
\hat{\psi}(u)_{n}=\frac{1}{n} \sum_{k=1}^{n}\left(1-e^{\Psi(\omega, u)_{k}}\right),
$$

and the two-sided $99 \%$ confidence interval of the estimator can be written as

$$
\left(\max \left[\hat{\psi}(u)_{n}-\frac{2.81}{\sqrt{n}} \sigma_{n}, 0\right], \min \left[\hat{\psi}(u)_{n}+\frac{2.81}{\sqrt{n}} \sigma_{n}, 1\right]\right),
$$




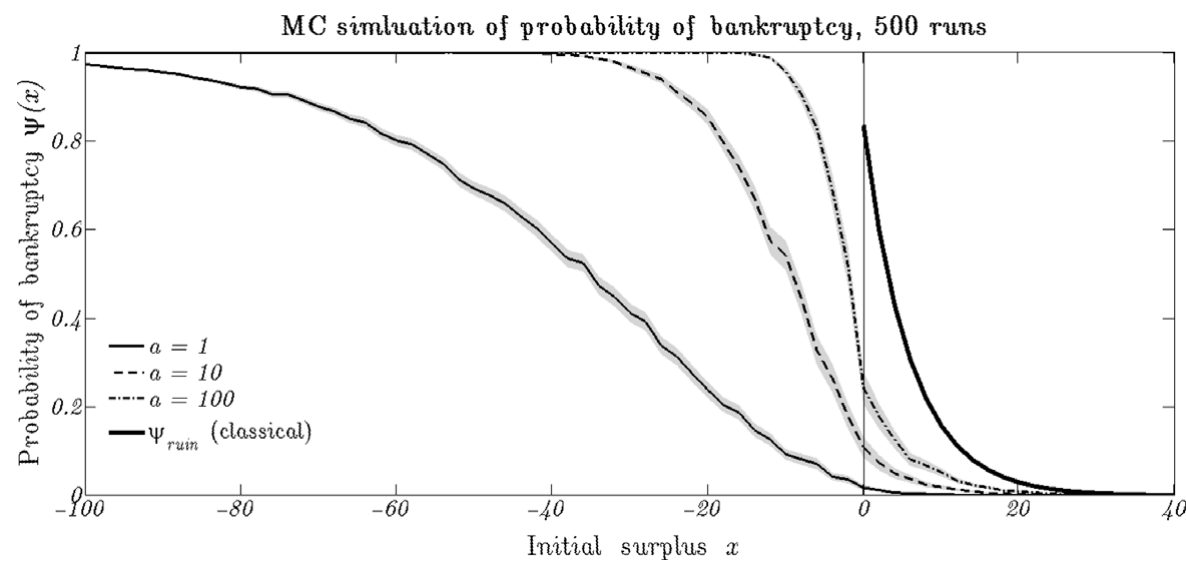

FIGURE 8: Simulation of $\psi(u)$ with $\omega_{2}(x)=-a x .500$ runs, shaded areas are the $99 \%$ confidence intervals.

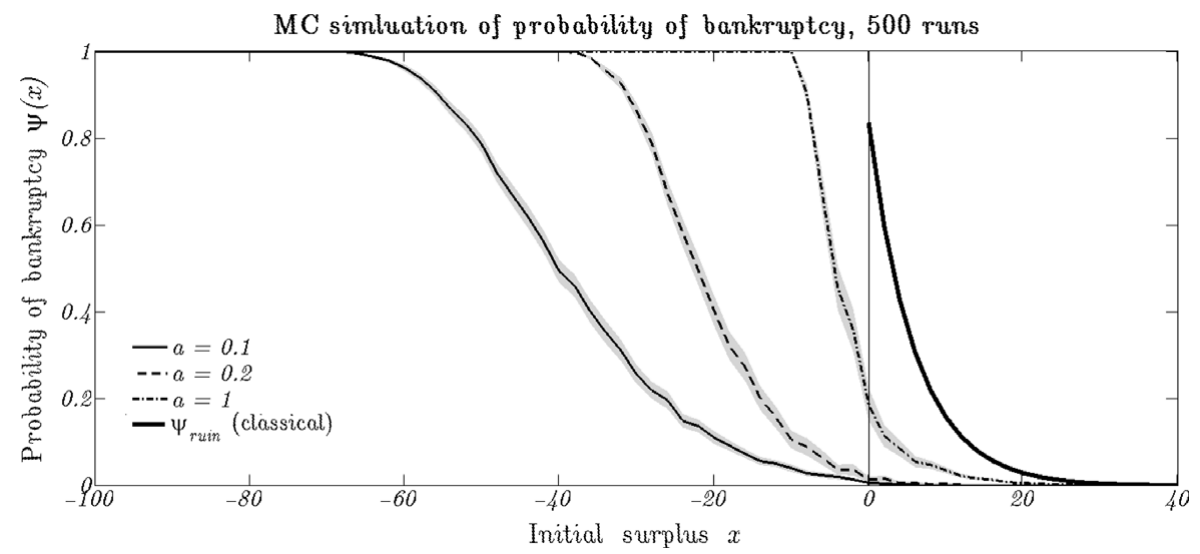

FIGURE 9: Simulation of $\psi(u)$ with $\omega_{3}(x)=e^{-a x} .500$ runs, shaded areas are the $99 \%$ confidence intervals.

with $\sigma_{n}=\sqrt{\frac{1}{n-1} \sum_{k=1}^{n}\left(1-e^{\Psi(\omega, u)_{k}}-\hat{\psi}_{n}(u)\right)^{2}}$, such that the bounds of the confidence interval converge to $\hat{\psi}(u)_{n}$ for $n \rightarrow \infty$.

3.3.2. Results. In order to underline the fast speed of convergence, the above simulation algorithm is executed based on only 500 sample paths. This already gives a solid approximation of the exact bankruptcy probabilities, which is illustrated in Figures 8 and 9 for the cases of linear and exponential bankruptcy functions. The curves depict the estimators of the bankruptcy probabilities for various choices of parameter $a$, and the shaded areas around those lines indicate the $99 \%$ confidence intervals based on the simulation outcome. When the number of sample paths is increased to only 1,000, the confidence intervals are no longer visible and the curves display the smooth shapes of the exact 
solutions. This illustrates the effectiveness of the two involved variancereduction methods.

Remark. The application of the above Monte Carlo method is not limited to the case of exponential claims. In particular, for claim size distributions where the classical ruin probability is known and where one can efficiently simulate from the deficit-at-ruin distribution, the same procedure as above can be employed. To state an example, consider the case of phase-type claims with representation $(\boldsymbol{\alpha}, \mathbf{T})$. The ruin probability is then well-known to be $\psi_{\text {ruin }, p t}(x)=-\frac{\lambda}{c} \boldsymbol{\alpha} \mathbf{T}^{-1} \cdot e^{\left(\mathbf{T}-\lambda / c \mathbf{t} \alpha \mathbf{T}^{-1}\right) x} \mathbf{1}$ with $\mathbf{1}=(1, \ldots, 1)^{\prime}$ and $\mathbf{t}=-\mathbf{T} \mathbf{1}$ (cf. e.g. Asmussen and Albrecher, 2010, page 264), and the deficit-at-ruin $-D$ is

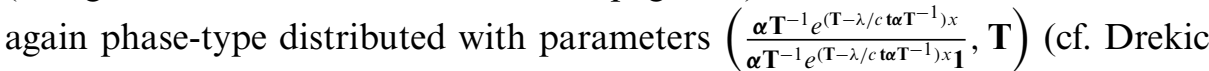
et al., 2004). Hence, the simulation for positive surplus levels can again be simplified by replacing the classical ruin probability in (45) by $\psi_{\text {ruin, } p t}\left(x_{+}\right), D$ can be sampled from the appropriate phase-type distribution and (46) does not depend on the claim size distribution.

\section{DISCUSSION OF THE DISCOUNTED PENALTY FUNCTION}

The analytical approach for the probability of bankruptcy can be extended to study more general quantities of the risk process. In this section, we will illustrate this for the discounted penalty function at bankruptcy

$$
m_{\delta}(x)=\mathbb{E}\left[e^{-\delta \tau} w\left(\left|C_{\tau}\right|\right) \mathbb{1}_{\{\tau<\infty\}} \mid C_{0}=x\right],
$$

where $\delta \geq 0$ is a discount force and $w(\cdot)$ is a function of the bankruptcy deficit at time $\tau$ (note that the surplus immediately before bankruptcy coincides with the bankruptcy deficit and, hence, does not need to be considered separately). For $\delta=0, w=1$ the discounted penalty function reduces to the bankruptcy probability $\psi(x)$.

Proceeding as in Section 2, one derives the following extensions of (6) and (7), as we distinguish by negative and positive surplus levels $x$ :

$$
\begin{aligned}
x \geq 0: 0= & c m_{\delta}^{\prime}(x)-(\delta+\lambda) m_{\delta}(x)+\lambda \int_{0}^{\infty} m_{\delta}(x-y) d F_{Y}(y), \\
x<0: 0= & c m_{\delta}^{\prime}(x)-(\delta+\lambda+\omega(x)) m_{\delta}(x)+\omega(x) w(-x) \\
& +\lambda \int_{0}^{\infty} m_{\delta}(x-y) d F_{Y}(y),
\end{aligned}
$$

with continuity at $x=0$ and more generally for all $x \in \mathbb{R}$ (also, the derivative $m_{\delta}^{\prime}(x)$ exists at all continuity points of $\left.\omega(x)\right)$. Using

$$
m_{\delta}(x)= \begin{cases}m_{\delta, u}(x) & \text { for } x \geq 0 \\ m_{\delta, l}(x) & \text { for } x<0\end{cases}
$$


gives

$$
\begin{aligned}
x \geq 0: 0= & c m_{\delta, u}^{\prime}(x)-(\delta+\lambda) m_{\delta, u}(x)+\lambda\left(\int_{0}^{x} m_{\delta, u}(x-y) d F_{Y}(y)\right. \\
& \left.+\int_{x}^{\infty} m_{\delta, l}(x-y) d F_{Y}(y)\right), \\
x<0: 0= & c m_{\delta, l}^{\prime}(x)-(\delta+\lambda+\omega(x)) m_{\delta, l}(x)+\omega(x) w(-x) \\
& +\lambda \int_{0}^{\infty} m_{\delta, l}(x-y) d F_{Y}(y),
\end{aligned}
$$

with $m_{\delta, u}(0)=m_{\delta, l}(0)$.

As the claim sizes are assumed i.i.d. exponential here, i.e. $f_{Y}(y)=v e^{-v y}$, we can eliminate the integrals by applying the operator $\left(\frac{d}{d x}+v\right)$ to $(52)$ and (53), and we arrive at the following system of linear differential equations,

$$
\begin{aligned}
x \geq 0: 0= & c m_{\delta, u}^{\prime \prime}(x)+(v c-(\delta+\lambda)) m_{\delta, u}^{\prime}(x)-v \delta m_{\delta, u}(x), \\
x<0: 0= & c m_{\delta, l}^{\prime \prime}(x)+(v c-(\delta+\lambda+\omega(x))) m_{\delta, l}^{\prime}(x) \\
& -\left(\omega^{\prime}(x)+v(\delta+\omega(x))\right) m_{\delta, l}(x) \\
& +\left(\omega^{\prime}(x) w(-x)-\omega(x) w^{\prime}(-x)+v \omega(x) w(-x)\right) .
\end{aligned}
$$

For $x \geq 0,(54)$ has constant coefficients and one obtains

$$
m_{\delta, u}=A e^{-R x}+B e^{\rho x},
$$

with $A, B \in \mathbb{R}$, where $-R<0$ and $\rho>0$ are the solutions to the characteristic equation

$$
\xi^{2}+\left(\nu-\frac{\delta+\lambda}{c}\right) \xi-\frac{\nu \delta}{c}=0 .
$$

For general functions $\omega(x)$ it is not straightforward to obtain a solution to (55) with $x<0$, as functions of $\omega(x)$ appear both in the coefficients of the homogeneous equation and in the inhomogeneous term. In the following, we will only discuss the case of approximating $\omega(x)$ by piecewise constant functions and penalty function $w(x)=e^{-q x}, q \geq 0$, which leads to quite tractable expressions.

\subsection{Piecewise constant bankruptcy rate functions}

Using (34), (55) can be re-written as a system of $n$ differential equations, describing the local dynamics depending on the value of $x$,

$$
\begin{aligned}
x_{k-1}<x<x_{k}: 0= & c m_{\delta, k}^{\prime \prime}(x)+\left(v c-\left(\delta+\lambda+\omega_{k}\right)\right) m_{\delta, k}^{\prime}(x)-v\left(\delta+\omega_{k}\right) m_{\delta, k}(x) \\
& +\omega_{k} e^{q x}(\nu+q) .
\end{aligned}
$$


Each differential equation has now constant coefficients so that we solve

$$
m_{\delta, k}(x)=A_{k} e^{-r_{k} x}+B_{k} e^{\rho_{k} x}+C_{k} e^{q x}, x_{k-1}<x<x_{k},
$$

where $-r_{k}<0$ and $\rho_{k}>0$ are the solutions of the characteristic equation

$$
\xi^{2}+\left(v-\frac{\delta+\lambda+\omega_{k}}{c}\right) \xi-\frac{v\left(\delta+\omega_{k}\right)}{c}=0
$$

$A_{k}$ and $B_{k}$ are to be determined constants, and

$$
C_{k}=-\frac{\omega_{k}(q+v)}{c q^{2}+\left(v c-\left(\delta+\lambda+\omega_{k}\right)\right) q-v\left(\delta+\omega_{k}\right)}=-\frac{\omega_{k}(q+v)}{c\left(q+r_{k}\right)\left(q-\rho_{k}\right)},
$$

results from finding an inhomogeneous solution to the equation.

We now have to find the constants $A, B, A_{k}, B_{k}$, for $1 \leq k \leq n$.

Boundary conditions. The upper boundary condition $\lim _{x \rightarrow \infty} m_{\delta, u}(x)=0 \mathrm{im}-$ plies $B=0$. As a lower boundary condition, we find

$$
\lim _{x \rightarrow-\infty} m_{\delta, 1}(x)= \begin{cases}0 & \text { for } q>0, \\ \frac{\omega_{1}}{\omega_{1}+\delta} & \text { for } q=0\end{cases}
$$

since $\lim _{x \rightarrow-\infty} m_{\delta, 1}(x)=\mathbb{E}\left[e^{-\delta \tau} \mathbb{1}_{\{\tau<\infty\}} \mid U(0)=-\infty\right]$ simply describes the Laplace transform of the time of bankruptcy which is the first epoch of the Poisson process with intensity $\omega_{1}$. $\operatorname{As}_{\lim _{x \rightarrow-\infty}} m_{\delta, 1}(x)$ is finite, we have $A_{1}=0$.

Continuity and IDE conditions. Imposing continuity at the $x_{k}$ values yields the conditions

$$
A_{k} e^{-r_{k} x_{k}}+B_{k} e^{\rho_{k} x_{k}}+C_{k} e^{q x_{k}}=A_{k+1} e^{-r_{k+1} x_{k}}+B_{k+1} e^{\rho_{k+1} x_{k}}+C_{k+1} e^{q x_{k}},
$$

for $1 \leq k \leq n-1$, and

$$
A_{n}+B_{n}+C_{n}=A
$$


Substituting the general form of $m_{\delta, k}(x)$, as derived in (56), into (53) produces $n$ more conditions. For $x_{k-1}<x<x_{k}$ and $1 \leq k \leq n$, we find

$$
\begin{aligned}
0= & c\left(-r_{k} A_{k} e^{-r_{k} x}+\rho_{k} B_{k} e^{\rho_{k} x}+q C_{k} e^{q x}\right) \\
& -\left(\delta+\lambda+\omega_{k}\right)\left(A_{k} e^{-r_{k} x}+B_{k} e^{\rho_{k} x}+C_{k} e^{q x}\right) \\
& +\omega_{k} e^{q x}+\lambda \int_{0}^{x-x_{k-1}}\left(A_{k} e^{-r_{k}(x-y)}+B_{k} e^{\rho_{k}(x-y)}+C_{k} e^{q(x-y)}\right) v e^{-v y} d y \\
& +\lambda \sum_{j=1}^{k-1} \int_{x-x_{k-j}}^{x-x_{k-j-1}}\left(A_{k-j} e^{-r_{k-j}(x-y)}+B_{k-j} e^{\rho_{k-j}(x-y)}+C_{k-j} e^{q(x-y)}\right) v e^{-v y} d y .
\end{aligned}
$$

The above equation can be re-written by using

$$
-c r_{k}-\left(\delta+\lambda+\omega_{k}\right)+\frac{\lambda v}{v-r_{k}}=0, \quad c \rho_{k}-\left(\delta+\lambda+\omega_{k}\right)+\frac{\lambda v}{v+\rho_{k}}=0
$$

and

$$
\omega_{k}+C_{k}\left(c q-\left(\delta+\lambda+\omega_{k}\right)+\frac{\lambda v}{v+q}\right)=0
$$

and after evaluating the integral expressions, we find, for $2 \leq k \leq n$, that

$$
\begin{aligned}
0= & \lambda v e^{-v x}\left[-\frac{A_{k}}{v-r_{k}} e^{x_{k-1}\left(v-r_{k}\right)}-\frac{B_{k}}{v+\rho_{k}} e^{x_{k-1}\left(v+\rho_{k}\right)}-\frac{C_{k}}{v+q} e^{x_{k-1}(v+q)}\right. \\
& +\sum_{j=2}^{k-1}\left(\frac{A_{j}}{v-r_{j}}\left(-e^{x_{j-1}\left(v-r_{j}\right)}+e^{x_{j}\left(v-r_{j}\right)}\right)+\frac{B_{j}}{v+\rho_{j}}\left(-e^{x_{j-1}\left(v+\rho_{j}\right)}+e^{x_{j}\left(v+\rho_{j}\right)}\right)\right. \\
& \left.\left.+\frac{C_{j}}{v+q}\left(-e^{x_{j-1}(v+q)}+e^{x_{j}(v+q)}\right)\right)+\frac{B_{1}}{v+\rho} e^{x_{1}(v+\rho)}+\frac{C_{1}}{v+q} e^{x_{1}(v+q)}\right] .
\end{aligned}
$$

Similarly, we can rearrange the IDE (51) for $x \geq 0$ to yield

$$
\begin{aligned}
0= & \lambda v e^{-v x}\left[-\frac{A}{v-r}+\frac{A_{n}}{v-r_{n}}\left(1-e^{x_{n-1}\left(v-r_{n}\right)}\right)+\frac{B_{n}}{v+\rho_{n}}\left(1-e^{x_{n-1}\left(v+\rho_{n}\right)}\right)\right. \\
& +\frac{C_{n}}{v+q}\left(1-e^{x_{n-1}(v+q)}\right) \\
& +\sum_{j=2}^{k-1}\left(\frac{A_{j}}{v-r_{j}}\left(-e^{x_{j-1}\left(v-r_{j}\right)}+e^{x_{j}\left(v-r_{j}\right)}\right)+\frac{B_{j}}{v+\rho_{j}}\left(-e^{x_{j-1}\left(v+\rho_{j}\right)}+e^{x_{j}\left(v+\rho_{j}\right)}\right)\right. \\
& \left.\left.+\frac{C_{j}}{v+q}\left(-e^{x_{j-1}(v+q)}+e^{x_{j}(v+q)}\right)\right)+\frac{B_{1}}{v+\rho} e^{x_{1}(v+\rho)}+\frac{C_{1}}{v+q} e^{x_{1}(v+q)}\right] .(60)
\end{aligned}
$$


Noting that $\lambda v e^{-v x}>0$, we can simplify the conditions as in (59) and (60) as follows to have, for $1 \leq k \leq n-1$,

$$
\begin{aligned}
& A_{k} \frac{e^{-r_{k} x_{k}}}{v-r_{k}}+B_{k} \frac{e^{\rho_{k} x_{k}}}{v+\rho_{k}}-A_{k+1} \frac{e^{-r_{k+1} x_{k}}}{v-r_{k+1}}-B_{k+1} \frac{e^{\rho_{k+1} x_{k}}}{v+\rho_{k+1}} \\
& =C_{k+1} \frac{e^{q x_{k}}}{v+q}-C_{k} \frac{e^{q x_{k}}}{v+q},
\end{aligned}
$$

and

$$
A_{n} \frac{1}{v-r_{n}}+B_{n} \frac{1}{v+\rho_{n}}-A \frac{1}{v-r}=-C_{n} \frac{1}{v+q} .
$$

All obtained conditions (57), (58), (61) and (62) can again be summarized by a linear equation system of the form

$$
U \cdot z=v
$$

for

$$
z^{\prime}=\left[B_{1}, A_{2}, B_{2}, \ldots, A_{n}, B_{n}, A\right],
$$

where

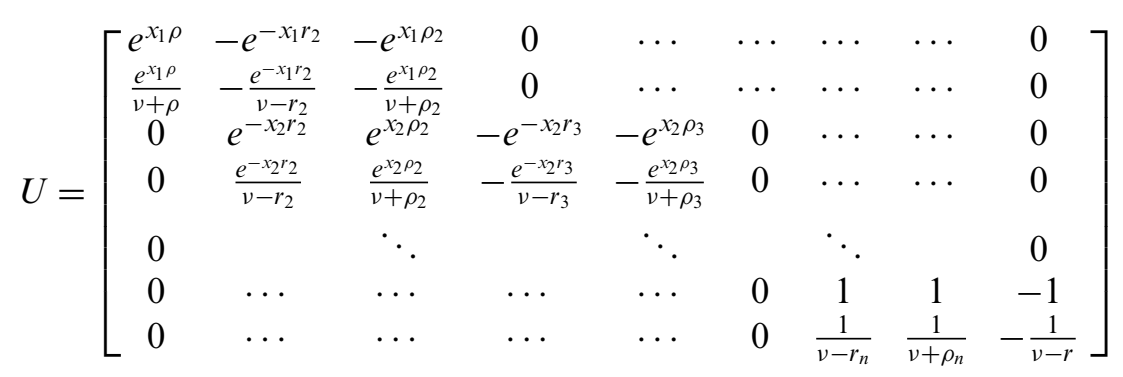

and

$$
\begin{aligned}
v^{\prime}= & {\left[e^{x_{1} q}\left(C_{2}-C_{1}\right), \frac{e^{x_{1} q}}{v+q}\left(C_{2}-C_{1}\right), \ldots, e^{x_{n-1} q}\left(C_{n}-C_{n-1}\right),\right.} \\
& \left.\times \frac{e^{x_{n-1} q}}{v+q}\left(C_{n}-C_{n-1}\right),-C_{n},-C_{n} \frac{1}{v+q}\right] .
\end{aligned}
$$

The discounted penalty function for $x>0$ (which would be the typical case of interest) then is

$$
m_{\delta}(x)=\left(U^{-1} v\right)_{2 n} e^{-R x},
$$

where the index indicates the $2 n$th component of the vector, and $U^{-1}$ is the inverse matrix of $U$. 


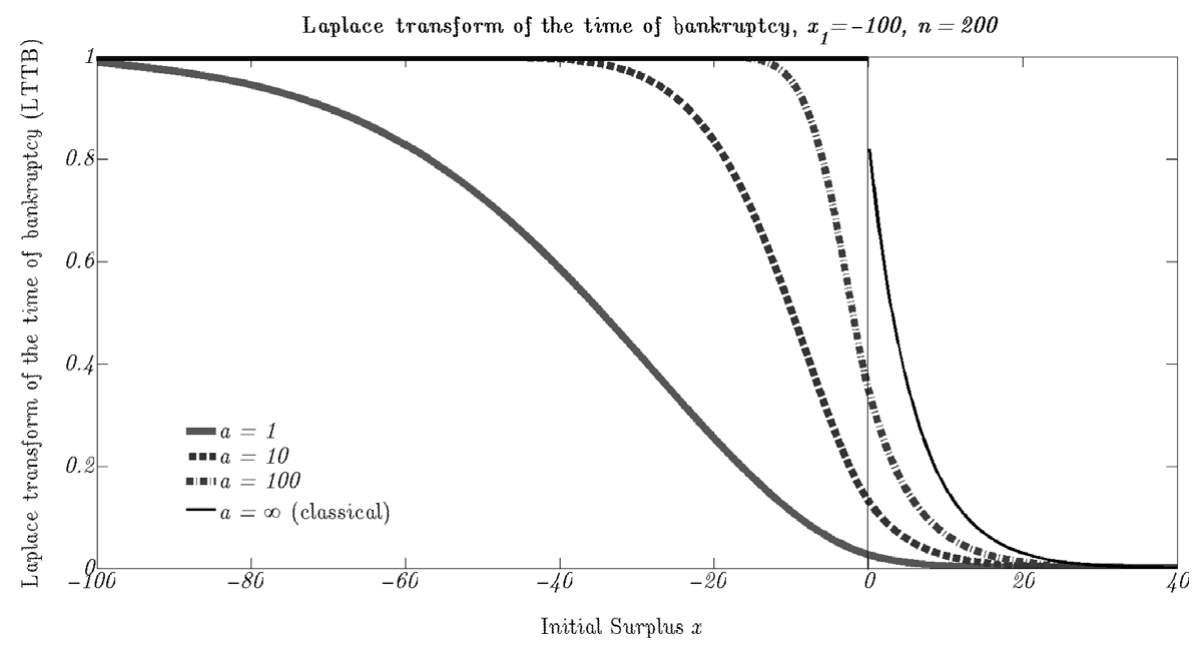

FIGURE 10: Laplace transform of the time of bankruptcy for linear $\omega_{2}(x)$.

The Laplace transform of the time of bankruptcy. We numerically illustrate the procedure for the Laplace transform of the time of bankruptcy $\mathbb{E}\left[e^{-\delta \tau} \mathbb{1}_{\{\tau<\infty\}} \mid C_{0}=x\right]$ (i.e. $q=0$ ). In the classical ruin case, one then has (see e.g. Dickson, 2005, p. 174)

$$
m_{\delta, \text { ruin }}(x)=\left(1-\frac{R_{\delta}}{v}\right) e^{-R_{\delta} x}
$$

where $-R_{\delta}<0$ is the negative solution of

$$
\xi^{2}+\left(\nu-\frac{\lambda+\delta}{c}\right) \xi-\frac{\nu \delta}{c}=0 .
$$

As in Section 3.2, linear and exponential bankruptcy rate functions are approximated by piecewise constant functions, and the linear equation system (63) is again solved using the Gauss-Seidel method. We choose $\delta=0.1$, and for the considered cases it turns out sufficient to set $x_{1}=-100$ and $n=200$. Figures 10 and 11 depict the resulting Laplace transform of the time of bankruptcy as a function of initial surplus. One observes that the shapes are similar to those of the probability of bankruptcy.

\section{CONCLUDING REMARKS}

In this paper, we considered a generalization of the classical ruin concept to a concept of bankruptcy, under which the surplus process can possibly continue despite temporary negative surplus, where the probability for having to indeed close the business increases, the more negative the surplus becomes. This 




FIGURE 11: Laplace transform of the time of bankruptcy for exponential $\omega_{3}(x)$.

amends a frequently raised critic of the classical ruin concept that crossing the surplus level 0 does not necessarily automatically mean ruin. The way in which this relaxed ruin concept is introduced provides some tractability. We showed in this paper that the resulting bankruptcy probability can be obtained explicitly in a Cramér-Lundberg model with exponential claims and certain types of bankruptcy functions. In addition, an approximation scheme with piecewise constant bankruptcy rates was worked out, which can approximate results for any bankruptcy rate function arbitrarily closely. Exploiting a link to occupation times, we also proposed an efficient simulation scheme, which may be used in much more general models as well. In particular, if the deficit distribution under the classical ruin concept is available, the simulation performance can be improved further. Finally, the results can be extended to more general quantities such as the bankruptcy deficit and the time of bankruptcy.

\section{ACKNOWLEDGEMENTS}

The authors would like to thank Hans U. Gerber for stimulating discussions on the topic, and two anonymous referees for helpful suggestions on the presentation of this paper. Support from the Swiss National Science Foundation Project 200021-124635/1 is gratefully acknowledged.

\section{REFERENCES}

Abramowitz, M. and Stegun, I. A. (1972) Handbook of Mathematical Functions, Applied Mathematics Series 55. Washington, DC: US State Department of Commerce. 
Albrecher, H., Cheung, E.C.K. and Thonhauser, S. (2011a) Randomized observation times for the compound Poisson risk model: Dividends. ASTIN Bulletin, 41(2), 645-672.

Albrecher, H., Cheung, E.C.K. and Thonhauser, S. (2011b) Randomized observation times for the compound Poisson risk model: The discounted penalty function. Scandinavian Actuarial Journal, in press, 10.1080/03461238.2011.624686.

Albrecher, H., Gerber, H.U. and ShiU, E.S.W. (2011) The optimal dividend barrier in the Gamma-Omega model. European Actuarial Journal, 1(1), 43-56.

Asmussen, S. and Albrecher, H. (2010) Ruin Probabilities, 2ND ED. World Scientific, Hackensack, New Jersey.

Chen, N., Glasser man, P., Nouri, B. and Pelger, M. (2012) CoCos, bail-in and tail risk. Office of Financial Research Working Paper No. 4 (2013), New York.

DAssios, A. and Embrechts, P. (1989) Martingales and insurance risk. Stochastic Models, 5, $181-217$.

DiCKSON, D.C.M. (2005) Insurance Risk and Ruin. Cambridge: Cambridge University Press.

DrekiC, S., Dickson, D.C.M., STANFORD, D.A. and Willmot, G.E. (2004) On the distribution of the deficit at ruin when claims are phase-type. Scandinavian Actuarial Journal, 2, 105-120.

Gerber, H.U. (1971) Der Einfluss von Zins auf die Ruinwahrscheinlichkeit. Mitteilungen der Schweizerischen Vereinigung der Versicherungsmatematiker, 71(1), 63-70.

Glasserman, P. and NOURI, B. (2010) Contingent capital with a capital-ratio trigger. SSRN report (abstract 1669686).

INSURANCE INFORMATION INSTITUTE (2012) Insolvencies/Guaranty Funds. Available at: www.iii.org.

LANDRIAUlt, D., RenAud, J.-F. and ZHOU, X. (2011) Occupation times of spectrally negative Lévy processes with applications. Stochastic Processes and Their Application, 121, 2629-2641.

MAES, S. and SCHOUTENS, W. (2010) Contingent capital: An in-depth discussion. SSRN report (abstract 1653877).

OXERA (2007) Insurance guarantee schemes in the EU. Commissioned by the European Commission (DG Internal Market and Services). Available at: ec.europa.eu/ internal_market/insurance/.

Schacht, J. W. and HePler, L.P. (2007) Insolvencies in-depth series. Risk and Insurance, Jan-Apr 2007 (in four parts). Available at: http://www.riskandinsurance.com/indepthdetail. jsp?storyId $=13404484$

ZHU, J. and YANG, H. (2008) Estimates for the absolute ruin probability in the compound Poisson model with credit and debit interest. Journal of Applied Probability, 45, 818-830.

HANSJÖRG ALBRECHER

Department of Actuarial Science, Faculty of Business and Economics, University of Lausanne, UNIL-Dorigny, CH-1015 Lausanne, and Swiss Finance Institute, Switzerland

E-Mail: hansjoerg.albrecher@unil.ch

VOLKMAR LAUTSCHAM (Corresponding author)

Department of Actuarial Science, Faculty of Business and Economics, University of Lausanne, UNIL-Dorigny, CH-1015 Lausanne, Switzerland

E-Mail:volkmar.lautscham@unil.ch 